\title{
Sense of agency for movements
}

\section{Christensen, Mark Schram; Grünbaum, Thor}

\section{Published in:}

Consciousness and Cognition

Link to article, DOI:

10.1016/j.concog.2018.07.002

Publication date:

2018

\section{Document Version}

Early version, also known as pre-print

Link back to DTU Orbit

Citation (APA):

Christensen, M. S., \& Grünbaum, T. (2018). Sense of agency for movements. Consciousness and Cognition, 65, 27-47. https://doi.org/10.1016/j.concog.2018.07.002

\section{General rights}

Copyright and moral rights for the publications made accessible in the public portal are retained by the authors and/or other copyright owners and it is a condition of accessing publications that users recognise and abide by the legal requirements associated with these rights.

- Users may download and print one copy of any publication from the public portal for the purpose of private study or research.

- You may not further distribute the material or use it for any profit-making activity or commercial gain

- You may freely distribute the URL identifying the publication in the public portal

If you believe that this document breaches copyright please contact us providing details, and we will remove access to the work immediately and investigate your claim 
Københavns Universitet

\section{Sense of agency for movements}

Christensen, Mark Schram; Grünbaum, Thor

Published in:

Consciousness and Cognition

DOI:

10.1016/j.concog.2018.07.002

Publication date:

2018

Document version

Early version, also known as pre-print

Document license:

CC BY-NC-ND

Citation for published version (APA):

Christensen, M. S., \& Grünbaum, T. (2018). Sense of agency for movements. Consciousness and Cognition, 65, 27-47. https://doi.org/10.1016/j.concog.2018.07.002 


\section{Sense of Agency for Movements}

Mark Schram Christensen ${ }^{1,2} \&$ Thor Grünbaum $^{3}$

1. Department of Neuroscience, University of Copenhagen, Denmark

2. DTU Compute, Department of Applied Mathematics and Computer Science, Technical University of Denmark, Denmark

3. Section of Philosophy, Department of Media, Cognition, and Communication, University of Copenhagen, Denmark

\section{Corresponding author:}

Mark Schram Christensen, MSc, PhD, Associate Professor

Department of Neuroscience

Panum Institute, Building 33.3

University of Copenhagen

Blegdamsvej 3B

DK-2200 Copenhagen $\mathrm{N}$

Denmark

markc@sund.ku.dk

\section{Conflicts of interest}

The authors declare no conflicts of interest. 


\begin{abstract}
In this paper, we argue that the comparator model is not a satisfactory model of sense of agency (SoA). We present a theoretical argument and experimental studies. We show 1) most studies of SoA neglect a distinction between SoA associated with movements (narrow SoA) and SoA associated with environmental events (broad SoA); 2) the comparator model emerges from experimental studies of sensory consequences narrowly associated with movements; 3) narrow SoA can be explained by a comparator model, but a motor signal model is simpler and explain narrow SoA equally well; and 4) standard experimental paradigms study only broad SoA. Finally, we present results from two experiments, where we have failed to induce illusory narrow SoA in healthy participants. We believe our experimental approaches should have led to illusory SoA, if the comparator model of SoA was correct. The results challenge proponents of the comparator model of narrow SoA.
\end{abstract}




\section{Introduction}

Many cognitive scientists accept that agents experience a sense of agency (SoA) when they perform voluntary actions. SoA has often been claimed to play important roles in motor control and motor cognition (Haggard 2017; Jeannerod 2006; Longo \& Haggard 2009; Moore et al. 2010). For many years, the dominant model to explain these experiences and functional roles has been the so-called comparator model (Frith 1987, Frith 2015). In this paper, we argue that the comparator model is highly problematic as a model of SoA. Our argument is partly theoretical and partly experimental.

Theoretically, we show that researchers in their characterisations of the SoA often shift from describing SoA as associated with bodily movements to describing it as associated with the planned consequences of the movements (e.g. auditory or visual events) (sections 2). Researchers often shift between understanding SoA in terms of control of bodily movements and in terms of control of external events. This shift is problematic for two reasons. First, the comparator model is a model of motor control. At its core, it comprises a mechanism or module that uses motor signals to compute likely proprioceptive feedback and feedback related to eye movements. It is problematic to cast this module in the role of a mechanism for predicting all kinds of likely sensory consequences (section 3). Second, even insisting that the comparator model for SoA is primarily a model for the SoA associated with movements (and not their consequences) turns out to be problematic. Reviewing the experimental literature shows that all standard SoA-paradigms are paradigms for studying the feeling of control over external events (section 4).

Experimentally, we test the claim that SoA for movements is produced by a comparator mechanism (section 5). Given the comparator model of SoA, we can predict situations in which agents should experience illusory SoA. That is, there should be situations in which agents are passively moved but it feels to them as if they moved voluntarily. Using a new paradigm, we test this prediction. The experiments show no illusion effects. To be sure, null results do not demonstrate anything; but the results nevertheless pose a serious challenge to proponents of the comparator model of explaining why inducing illusory SoA for movements is so difficult.

Taken together, the theoretical and experimental arguments show that the comparator model of SoA is highly problematic (section 6). The near absence of paradigms to study the SoA for movements has made it difficult to test the comparator model and develop alternative proposals. One proposal that would explain the results of the experiments presented in this paper would be a model according to which SoA for movements can be driven primarily by motor signals. 


\section{SoA for movements}

A persistent form of confusion creates problems for neuro-cognitive research on SoA. In this section, we illustrate a prominent version of this confusion. In a recent review, Haggard characterises SoA in the following way:

The sense of agency is the feeling of making something happen. It is the experience of controlling one's own motor acts and, through them, the course of external events. [...] the experience that occurs before, during and after actual muscular movement, rather than on beliefs or facts about potential actions. Thus, I use the term sense of agency to refer to an experience that accompanies the performance of a specific motor act. (Haggard, 2017, p. 197) Two different ideas are manifest in Haggard's definition of SoA. First, SoA is tied to specific motor acts. Here the idea is that a specific kind of feeling of activity is accompanying my voluntary movements but not the involuntary ones. Second, SoA is characterised as a broader phenomenon that refers to an experience that covers the complete temporal stretch of the action: preparation, motor performance, and environmental consequences. So, not only is SoA a strictly movement-related phenomenon, it is also the feeling an agent might have that she is the one controlling the occurrence of some environmental event (say, making a doorbell ring or turning on a light by pressing a button).

A confusion is created when these two ideas in the characterisation of SoA are coupled with one common, simple explanatory mechanism. The comparator model of motor control offered a simple way to explain the movement-related SoA. Often without noticing, this model of motor control has been extended also to the explanation of the feeling of control over external events. In the next section, we will look more closely at the way in which the comparator model, originally developed to explain a set of motor related phenomena, has been used to explain also the feeling of control over

external events. For now, we will focus on the two ideas manifest in Haggard's characterisation of SoA.

In what follows, we will call the SoA narrowly associated with voluntary movements the narrow SoA and the broader notion of a feeling of control over environmental events the broad SoA. Narrow SoA is only related to the bodily movements, whereas broad SoA includes additional events in the environment that accompany the bodily movement (see Christensen \& Grünbaum, 2017, for a further description of this distinction). The distinction between the two becomes evident when the 
same bodily movement, for instance, the movement of one's finger, can lead to several different events in the environment, such as a light that turns on or a doorbell that rings.

The confusion consists in using the same motor control mechanism to explain both the narrow SoA and the broad SoA. This enlargement of scope often goes unnoticed for the reason that researchers often have been inattentive to the distinction between narrow and broad SoA. Some researchers define SoA in relation to movements (for instance, Lynn et al., 2014, p. 1: "[...] sense of agency, i.e., the intrinsic sensation of being in control of one's own actions." See also Gallagher, 2000, p. 15, Synofzik et al., 2013, p. 1). Some researchers emphasize the control of external events (for instance, Wen et al., 2016, p. 1: “The ubiquitous experience of a subjective feeling of control over the outcome of events through one's behaviour refers to sense of agency."). Finally, some researchers include both movements and external effects in their definition of SoA (for instance, Caspar et al., 2016, p. 585: "Sense of agency refers to the subjective experience of controlling one's actions, and, through them, external events”. See also Dewey \& Knoblich, 2014, p. e110118, Moore et al., 2012, p. 1748, Spaniel et al., 2016, p. 916). The important point here is not so much the differences in stressing movements or external events in the definitions of SoA but the fact that the distinction between narrow and broad SoA has gone unnoticed and that both aspects of SoA are coupled with the same explanatory mechanism (i.e., the comparator mechanism), as will become apparent in the next section.

As is common, Haggard (2017) distinguishes between, on the one hand, SoA understood as an experience or low-level phenomenal feeling and, on the other hand, beliefs or judgements about one's action and its environmental consequences (see Synofzik et al., 2008). The above-mentioned confusion is not a neglect of this distinction between sense of agency (SoA) and judgements of agency (JoA). The confusion concerns only the definition and explanation of SoA. It is with respect to the standard characterisation of SoA that researchers have neglected to acknowledge the distinction between narrow and broad SoA.

Our focus in the present paper is the narrow SoA, i.e. the SoA associated with the performance of specific motor acts (rather than distal consequences of movements). There are reasons for thinking that the performance of voluntary movements is associated with a distinctive kind of experience. When I place my hand on the table in front of me and lift my index finger, I have an experience of moving my finger upwards. Such simple examples make it intuitively appealing that there is a sense of activity directly related to one's bodily movement. The extent to which this experience is clear may depend on the amount of attention paid to the movements. These intuitions are corroborated by 
experimental evidence suggesting that there is a distinct type of experience associated with motor performance. These studies have either used direct electrical cortical stimulation during surgery (Desmurget et al., 2009; Fried et al., 1991) or indirect magnetic cortical stimulation (Amassian, Cracco, \& Maccabee, 1989; Christensen et al., 2010) using transcranial magnetic stimulations. These studies suggest that participants feel a sensation of movement or urge to move directly related to the movement (for discussion, see Christensen \& Grünbaum, 2017).

\section{The comparator model}

The dominant explanation of both narrow and broad SoA is the so-called comparator model. In this section, we trace the explanatory origin of the model. The model was developed to explain a set of sensory phenomena narrowly related to movements and movement control. The model has only recently been extended to explain also SoA. In its simplest form, the comparator model of SoA explains SoA as an experience that arises when a comparison between the predicted sensory feedback and the actual sensory feedback of a voluntary movement shows the two feedback signals to be equal. The central assumption of the model is that the sensory predictions are computed from copies of motor commands. As will become clear, when the model is used to explain broad SoA, it is assumed that external sensory consequences can be predicted solely based on motor signals. However, in order to understand how the comparator model has ended up as being the dominant model for SoA, we will take a step back in time to look at the development of the comparator model principle. This development is summarised in Figure 1.

\subsection{The first indirect evidence of a comparator mechanism}

Von Holst and Mittelstaedt (1950) and Sperry (1950) were the first to propose the existence of a comparator mechanism to cancel out the sensory effects caused by one's own movements. Developed independently, the two papers indirectly infer that a mechanism must exist that cancels the sensory effects that an organism's own movements exert on its own sensory organs. Von Holst and Mittelstaedt describe the cancellation as an arithmetic operation (addition) of a positive efference signal (the efference copy, EC) and a negative afferent (A) signal, which, when equal (A=EC), would cancel out each other (see Figure 1A). When $\mathrm{EC} \neq \mathrm{A}$, the error signal is processed further upstream of the CNS and can be used to modulate the efferent command signals.

Sperry (1950) coined the term corollary discharge (CD), but there are considerable inconsistencies in the terminology used with respect to EC and CD, something McCloskey (1981) 
describes in more detail. He uses the following distinctions: "It must be stressed that efference copy, as envisaged by von Holst, is unlike corollary discharges in two important ways. First, the efference copy is required to cancel the reafference exactly, whereas corollary discharges provide internal adjustment of sensory centers without cancellation of reafference necessarily occurring. Second, in contrast to corollary discharges proposed specifically to deal with perceptual stability, efference copy is proposed simply as a "mechanism for distinguishing reafference from exafference"" (McCloskey 1981, p. 1419). McCloskey continues: "[...] the term corollary discharges should be reserved for those internal consequences of motor commands that affect sensations, either by central modification of the processing of the sensory signals generated peripherally or by their own independent actions." (McCloskey 1981, p. 1420). In the remaining part of this paper, we will use thesedistinctions between EC and CD as proposed by McCloskey. But as we will see in Figure 1, these distinctions are not used consistently.

In 1966, Donald M. MacKay proposed a slightly different model of the comparator mechanism including feedforward signals (see Figure 1B top). According to MacKay, the idea of a cancellation is problematic because the accuracy required for an exact cancellation would have to be extremely high (MacKay, 1966). Together with Mittelstaedt, MacKay (MacKay \& Mittelstaedt, 1974) refines the interpretation of the original proposal of the EC mechanism and the functional role of the comparator mechanism (see Figure 1B bottom). They argue that:

[...] what the C.N.S. must do is not to eliminate the sensory reafference produced by exploratory movement, but to evaluate it. Appropriately evaluated, it offers positive evidence of the stability of the explored world. Information from motor centres would on this view be required only to maintain appropriate central criteria of evaluation rather than to generate exact efferent copies for subtraction from the reafferent signal (MacKay \& Mittelstaedt, 1974, p. 72).

Contrary to the original proposal of the EC principle, MacKay argues that the controller system does not play the role of sensory cancellation because, if that was the case, a movement in a perfectly stable environment, which was perfectly predicted, would lead to no perception of movement in that environment (see Figure 1B bottom). He uses the example of touching a uniform surface: When we move our finger, it is not the case that we do not feel the surface, as the sensory cancellation idea would predict, rather we get a confirmation that the surface is stationary. The same goes for voluntary eye movements. If the evaluator were to cancel the sensory feedback with the 
predicted feedback, it would require immense accuracy of the model compared with the combined accuracy of the performed movement and predictability of the sensory feedback.

What is evident from the models formulated by MacKay $(1956,1966,1978)$ (see also Figure 1B), is that none of them incorporates a signal of a predicted sensory consequence of a movement, but rather a comparison between intended states and sensory states, where the comparator error can be used to adjust the movement. This serves very much the same purpose as Renshaw and gamma control reviewed by Roland (1978) and displayed in Figure 1C, although with the finer adjustments taking place at spinal levels, whereas the proposal by MacKay is unspecific in terms of neuronal implementation.

To cut a long story short, EC signals were initially identified for eye movements as a mean to make a cancellation of the effect of the organisms own movements, but the functional role of these signals was revised, and their possible role was suggested to be stabilization of the visual scene during eye movements (MacKay \& Mittelstaedt, 1974; Jeannerod et al., 1979; Bridgman, 2010).

\subsection{Comparator models in relation to schizophrenia}

Until now no specific mentioning of the comparator mechanism in relation to SoA is to be found in the literature. This changes in 1978, when Feinberg (Feinberg, 1978) proposes that patients with schizophrenia may have troubles in discriminating which movements are externally generated and which are internally generated. Feinberg mentions the feedforward proposal by MacKay from 1966 which Feinberg denotes internal feedback, i.e. not as a cancellation mechanism but as a monitoring principle. If this internal feedback for some reason becomes inappropriate, it can lead to delusions. As a final conclusion, Feinberg explains that the internal feedback or CD signals may play a role in distinguishing between self- and externally generated movements, and furthermore they may allow monitoring of efferent motor commands before the effector response has occurred. Both of these two possible functions of the internal feedback (the CD signals) are closely related to the sense of agency.

In 1987, Frith (Frith, 1987) proposes that monitoring of actions comprise monitoring of willed intentions (WI), stimulus intentions in response to environmental demands (SI), and finally selected actions, which allow for rapid error detection. This proposal was made as a part of his explanation of positive and negative symptoms in schizophrenia. According to Frith's model, positive symptoms, such as hallucinations, delusions and agitated movements (based on National Institute of Mental Health's definitions), are present when "[...] willed intentions lead to actions, but these willed intentions are not monitored correctly" (p. 631). By contrast, negative symptoms, such as reduced 
emotional expression, reduced feeling of pleasure and difficulty in initiating and maintaining activities, arise when "intentions of will are no longer properly formed and so actions are rarely elicited via this route." (p. 631). Frith's model is displayed in Figure 1D as a composite of two different models with partly overlapping elements.

The proposed system can distinguish between the following three conditions. 1) Stimulus error, which is "a response that is appropriate to an immediate stimulus, but is not appropriate to [...] current goals and plans" (p. 636), such as washing your hands in the bath room, when your actual plan was to brush your teeth. 2) Willed errors, which are errors where the agent "based his action on his wishes and expectations rather than the appropriate stimulus", for instance, when two people meet in a narrow passage and both step to the same side because they believe the other would have done differently. 3) Meaning errors, which occur when "we have made the response we intended and have failed because we did not know the meaning of the stimulus", for instance, pushing a door with a sign saying "ziehen" (German for pulling). These three different types of error comprise the outcome of what later is going to be known as the comparator model. It is interesting to note that the central monitor proposed by Frith incorporates three types of information, which can be compared interchangeably giving rise to different comparisons and hence error signals.

Building on von Holst, Mittelstaedt, and MacKay's work on comparator mechanisms, Jeannerod and colleagues have explored action-attribution, which is the process of determining whether an action is made by oneself or another agent. Daprati et al. (1997) investigated agency in patients with schizophrenia and showed that patients with positive symptoms tended to misattribute alien hands to themselves. Maybe the first explicit discussion of the problem of determining who is the agent is in Georgieff \& Jeannerod (1998). Georgieff and Jeannerod explicitly mention the EC principle of von Holst and Mittelstaedt (1950) as a comparator. Central to their argument is the hypothesis that pathologies of action-attribution (such as delusions of control in schizophrenic patients with psychosis) can be explained in terms of "a dysfunction of the comparison process" (Georgieff \& Jeannerod, 1998, p. 474).

\subsection{Motor learning and sensory attenuation}

Simultaneous with the development of the comparator model as an explanation of problems of selfother discrimination and delusions in schizophrenia, another line of research evolved from a completely different perspective. This is the concept of internal models and forward models. The idea of internal models arose from network descriptions of the cerebellum (Marr, 1969; Ito, 1970; Albus, 
1971), which fostered the idea of a computational network model that could explain motor learning (Kawato et al., 1987) (see Figure 1E), in particular supervised learning (Jordan \& Rumelhart, 1992) (see Figure 1F). The concept of an internal model comprises two distinct functional models: The forward model (Figure 1F top) that uses an efference copy to make a prediction of the sensory consequences of the movement, and the inverse model (Figure 1F bottom) that computes a description of how the agent comes from its present state to the desired state. The output from the forward model, i.e. the prediction of the sensory feedback, can be compared with the actual feedback, giving rise to supervised feedback learning and cancellation of sensory re-afference (Miall \& Wolpert 1996) (see Figure 1G). The output (i.e. motor commands and efference copy signals, EC) from inverse models can be used to make online error correction using state estimates of the body in the absence of sensory feedback (Miall \& Wolpert 1996). The use of EC signals for online error correction was also suggested by Angel (1976). However, in particular state estimation seems to be important in the internal models (Wolpert et al. 1995). By the mid-90s, the direct association of the comparator mechanism and SoA had not yet been proposed.

By the end of the 1990s, Chris Frith begins collaborating with Daniel Wolpert and Susan Blakemore in order to explain the phenomenon of sensory cancellation of self-produced tactile stimuli. Blakemore et al. (1998) argue that the principle of cancellation of self-produced tactile stimuli can be derived directly from internal models:

By comparing this prediction with the actual sensory feedback, it is possible to distinguish the sensory consequences of our movements from sensory signals due to changes in the outside world. These mechanisms have mainly been studied with reference to eye movements. However, it seems that sensory predictions produced in conjunction with the motor command are not restricted to eye movements, but also provide perceptual stability in the context of all self-produced actions; our ability to monitor, and recognize as our own, self-generated limb movements, touch and speech, suggests the existence of a more general mechanism. (p. 635). The first explicit mentioning of a single comparator mechanisms between predicted and actual sensory feedback leading to a specific perceptual phenomenon outlined as a graph model is by Blakemore et al (1999). According to this model, the predicted and actual sensory feedback is compared, and if there is a discrepancy, tactile stimuli will feel ticklish. The perceptual phenomenon of tickling is explained by a simple comparison between predicted and actual sensory feedback (see Figure 1H). This line of thought is perfectly in line with the original EC proposal as a cancellation mechanism, but note that it also incorporates the idea of perceptual stability, i.e. MacKay's argument, 
and involves all types of self-generated limb movements. Notice also that this model does not involve prediction of external events; it does, however, involve predictions of tactile events applied to the body through an action of the body applied to the body.

Returning to the explanation of abnormalities in awareness of actions, for instance delusions in schizophrenia, Frith et al. 2000 suggests that:

[...] in the presence of delusions of control, the patient is not aware of the predicted consequences of a movement and is therefore not aware of initiating a movement. [...] There is nothing obviously abnormal in the motor control of these patients. This suggests that accurate representations of predicted states are available and used by the motor system. However, these representations are not available to awareness. (p. 1784).

The simple model according to which predicted feedback is compared with actual sensory feedback is now revised to comprise a comparison of the predicted sensory feedback with both the actual sensory feedback and the desired state. Furthermore, the desired state is also compared with the actual state. It is important to note that until now it has been an implicit assumption that the comparisons are concerned with the sensory effects narrowly tied to movements, such as retinal stimulation, proprioceptive feedback, and tactile sensations.

\subsection{Sense of agency and the comparator model}

In the famous intentional binding experiment, Haggard et al. (2002) refer to the mechanisms proposed by Frith (1992/2012) and associate it with delusions of control in schizophrenia patients. According to Haggard:

The intentional binding process fits well with recent models of action-effect matching, both in neuropsychiatry and in computational motor control. Such matching processes typically induce motor learning, but also produce a characteristic conscious experience, particularly when a mismatch occurs. (Haggard et al., 2002, p. 384)

To our knowledge, the first time the sense of agency is explicitly associated with the comparator/forward model idea, is by Haggard (2005) (see Figure 1I). According to Haggard, it is “[...] an integration of efference, predicted feedback and sensory information, which might lead to the sense of agency" (Haggard,2005, p. 290, Figure text). Furthermore, it is proposed that "the sense that our actions cause effects in the outside world (so-called 'agency')" (p. 290). A similar proposal is made by Jeannerod (2006) in order to explain the self/other distinction (see Figure 1K). 
However, at the same time, Tsakiris \& Haggard (2005) propose that motor signals might be sufficient to produce SoA (see Figure 1J, black box with SoA in italics): "The sense of agency, which generates our experience of actions, seems to be efferent driven, and it can be thought of as the first crucial step towards the realisation of intentions" (Tsakiris \& Haggard 2005, p. 403). They continue: "This efferent copy seems to be sufficient condition for the raw sense of agency [...]. This bias might reflect the knowledge of the system that "it is me who is acting" as a direct consequence of an efferentdriven sense of agency," (Tsakiris \& Haggard, 2005, p. 404).

Here it is interesting to note that the raw sense of agency apparently can be realised by the motor command/efference copy alone without any comparison taking place (see Figure 1J). This idea seems to disappear from the subsequent discussions. At most, motor signals can play a modifying role. Subsequently, the comparator model is described in papers as the principle of SoA arising when there is a match between the predicted and actual sensory feedback (see both the formulation by Synofzik (2008) and David et al (2008) (Figure 1L \& 1M)). Recently, Haggard and Chambon (2012) have proposed that SoA is produced mainly by the outcome of a comparison between the predicted and actual sensory feedback, and that a direct signal from the inverse model (that bypass the efference copy) might strengthen or weaken the outcome (see Figure 1N). In the latest formulation of the model, Haggard states the following:

A forward model uses a copy of the current motor command (known as an efference copy) to predict the probable sensory consequences of the command. This prediction is compared with sensory feedback signals that provide information about the ongoing action and about its effects on the external environment. The result of the comparison can be used in three ways: to adjust the current motor command (1); to attribute agency for actions and environmental events (2) (if the comparator gives a result of zero then the event is caused by one's own action); and to attenuate predictable, self-produced sensations (3). (Haggard 2017, p. 202).

\section{[Insert Figure 1 here]}

We have also included the active inference model, as it is described in Adams et al (2013) (see Figure 10). Note how, according to this model, the interpretations of predictive signals, motor commands and prediction error signals are reversed. This is not a comparator model because the arrows have other roles than they would according to comparator models. 


\subsection{Discussion}

Let us now sum up this brief history of the development of the comparator model for SoA. The comparator mechanism was proposed for cancelling sensory effects caused by one's own eye movements (the reafferent signal), and directly related to internal sensory consequences of a movement. As was apparent from MacKay's arguments in the 1960s, it is difficult to explain how the CNS is able to compute sensory predictions from motor commands given the high degree of precision a comparison with feedback would seem to require. The alternative interpretation has been that the EC or CD signals are used to create stable perceptions of the surroundings.

Throughout the years, the comparator model has been turned into a mechanism that can account for predictions of distal effects in the external environment. This becomes apparent already with Frith's 1987-model (see Figure 1D) and in Haggard's later models (Figure 1I and 1N), where the feedback is unspecific with respect to modality. Given the demands on precision, the problems of how the forward model computes sensory predictions are serious enough when the comparison concerns predicted retinal changes and proprioceptive feedback.

The problem would seem to be much worse when dealing with prediction of external sensory consequences (for instance, when pressing a button can lead both to a doorbell ringing or a light turning on). One of the reasons for this is that if we compare the external consequences with the movements that produce them, the external consequences are usually delayed. Predicting external consequences requires that knowledge about the delays of mediating mechanisms can be incorporated into the comparator model. If I turn the key in my car, it takes a little while before the engine starts. Therefore, in order to make the prediction that my action (turning the key) will make the engine hum (i.e. start the engine), beliefs about how an engine works or prior experience with this type of action are required. Similarly, predictions about auditory events as a consequence of a button press build on prior beliefs about the relevant mechanism and their delays. It is a plausible assumption that a system using copies of motor commands to compute predictions of internal sensory feedback is cognitively insulated and modular. It is much less plausible that the same could be said of a system that computes predictions of external sensory events. It is unlikely that the mechanism computing a prediction of somatosensory and proprioceptive feedback from copies of motor signals is also able to predict the auditory event of the humming engine.

The current dominant models of SoA require the comparator mechanism to affirm predicted sensory consequences of internal as well as external sensory consequences. However, it is at present not clear how motor related signals directly can account for predictions of external events. There is 
the possibility that different internal models can account for different sensory transformations with respect to manipulations of visual feedback. Imamizu et al. (2004) showed how different internal models within the cerebellum could account for different manipulations (rotations and speed modifications) of the visual feedback. Consequently, for the same motor commands different forward and inverse models accounted for the visual manipulations. However, to generalize from this to all external events will require more evidence. It would require that we conduct experiments involving auditory stimuli, tactile stimuli, and complex sensory consequences. Ideally, it would require that an experiment is designed in such way that the same simple movement or action leads to different sensory consequences.

Using the model of a comparator mechanism deriving its sensory predictions from motor commands to explain the broad SoA therefore seems problematic. As long as we do not understand how the forward model computes predictions of external sensory events, the model provides us with no explanation of broad SoA. Our focus in the present paper is the narrow SoA. As an explanation of narrow SoA, the comparator model is more promising. The existence of associative mechanisms linking motor signals and sensory predictions is more plausible concerning retinal and proprioceptive stimulations. As we saw above, a survey of the literature shows that at least two different explanations of narrow SoA are possible. It is possible that the narrow SoA is generated by motor signals alone or that narrow SoA is generated by the comparison of predictions and actual feedback.

We can thus formulate two competing and equally probable models of narrow SoA. (1) The dominant comparator model according to which a comparator mechanism provides a positive or negative signal for SoA (see Figure 1M). As the difference between predicted and actual feedback goes towards zero, the signal becomes stronger. According to most versions of the comparator model, the signal for narrow SoA can be modulated in various ways (for instance, by the fluency of action selection, see Wenke et al., 2010). (2) The motor-signal model according to which motor commands provide a sufficient signal for narrow SoA (that could be a direct perception of the motor signal or perception of an efference copy). This signal can be modulated in various ways, for example by fluency signals and comparator signals (in case of error signals). In Figure 2, we have, based on the internal model proposed by Jeannerod (2006), illustrated two versions of the motor-signal model for narrow SoA. In Figure 2A, we propose a pure motor-signal model without depending on a comparator mechanism, and in Figure 2B, a model where SoA can be modulated by a prediction error from a comparator unit. One general question we would like to pursue is what additional information the 
model depicted in Figure 2B provides (by comparison with 2A) when there is a match between the predicted and actual sensory feedback.

\section{[Insert Figure 2 here]}

Our general claim is that the motor control models are open to the formulation of two competing models of narrow SoA: The simple comparator model and the motor-signal model. We need to design experiments that can distinguish between the predictions made by these models. This assessment of the comparator model for SoA is related to, yet different from, recent critical discussions in the philosophical literature.

The philosophical discussions seem to have been insufficiently attentive to the distinction between narrow and broad SoA. Pacherie (2008) argues that the internal model concept can be used at many different levels leading to a hierarchical model of distal intentions, proximal intentions and motor intentions with comparator mechanisms at many levels. According to Pacherie, one cannot use a single comparator to account for all aspects of SoA. Similarly, Shepherd (2015) argues that multiple levels of goals achieved during an action require multiple comparator loops. Using the hierarchical model of intentions and comparator modules or multiple comparator loops resembles some of the features of the active inference model, mentioned in the previous section. Carruthers (2012) argues that the comparator model of SoA needs to be adjusted more or less case by case/experiment by experiments, suggesting that it is not a sufficient model to describe SoA and that the multi-factorial weighting model of Synofzik et al. (2008) may be more appropriate. Finally, Mylopoulos (2012) discusses high-level, low-level and integration approaches to SoA and argues that both the low-level and integration approach account fail to fully explain SoA. In particular, she uses examples where both sensory attenuation, intentional binding and schizophrenia can be separated from forward modelling (or more generally internal models). Common to all these arguments is a neglect of the distinction between narrow and broad notions of SoA (see, however, Shepherd, 2016, and Mylopoulos, 2017. In particular Shephard, 2016, who proposes that merely trying can give rise to perceptual experiences similar to SoA without issuing a motor command. Whether this is compatible with our proposed model (Figure 2A) is not yet possible to determine).

Without describing all of the arguments of these authors, it is clear that a single comparator mechanism cannot fully account for all aspect of SoA, in particular, a simple comparison between the predicted and actual sensory feedback is not a satisfactory model of broad SoA (see Grünbaum, 
2015). More evidence is needed to decide whether a single comparison is a correct model of narrow SoA.

\section{Standard paradigms}

In this section, we defend the claim that the most common SoA paradigms study only broad SoA of consequences in the environment and not narrow SoA of movements. Experimental data from these paradigms can therefore not be used to determine what kind of signal is driving the narrow SoA and settle the question of which of the competing models is correct.

We (Christensen and Grünbaum, 2017) have recently reviewed the standard experimental paradigms more comprehensively. There are broadly speaking three types of experiment used to study SoA. First, some studies have used manipulations of the ongoing sensory feedback signal (typically visual). This is by far the most popular method to manipulate SoA. The classic example is the alien hand experiment (Nielsen, 1963) later computerised by Fourneret and Jeannerod (1998), where line drawing movements are transformed into a visually distorted version of the same line drawing movement (see also Ritterband-Rosenbaum et al., 2011,2012, 2014a, b). The distortions of visual feedback can either be of a spatial or temporal nature, i.e. angular deviations or delays. In one set of experiments, participants were asked to make a similarity judgement between their own motor performance and predefined lines (Fourneret and Jeannerod, 1998). In other variants of the paradigm different types of distortions have been employed such as bimanual in-phase anti-phase hand opening and closing (Fink et al., 1999), degree of rotation of visually presented image of hand (Farrer et al., 2003), and visuo-proprioceptive conflicts while performing mouse movements (Balslev et al., 2005, 2006). In these experiments, researchers measure SoA by anything from evaluating the strangeness of movement (Fink et al., 1999), reporting whether the displayed movement is one's own, a distorted version of one's movement or someone else's movement (Farrer et al., 2003), or using 0-100 point scales of sense of control to evaluate the subjective experience of the movement (Nahab et al., 2011). Common to all these studies is that participants are asked to focus on and report experiences with respect to the distal sensory feedback caused by the action.

Second, there exist studies of action-effect associations, in which the SoA is evaluated according to whether the participants feel that they produced some sensory consequence. One very influential study is Sato and Yasuda (2005). The authors induced SoA, using a simple button press experiment, where participants at their own pace freely pressed two buttons using their left or right index finger. The button press was immediately or with a delay followed by one of two tones. In a 
congruent task, the tone following button presses was always the same, in the incongruent task, the tones did not necessarily follow the same button press. Participants had to evaluate on a scale from 0 to 100 whether they agreed with "I was the one who produced the tone". In a very influential variant of the general action-effect paradigm, Haggard and colleagues have studied the effect of "intentional binding" (Haggard, Clark \& Kalogeras, 2002). The temporal binding phenomenon has been suggested as an implicit measure of SoA and has been used in many subsequent studies as an objective measure of SoA (Moore \& Obhi, 2012). It is not easy to tell what exactly is being measured by the intentional binding effect. The effect's relation to JoA has turned out to be opaque (Dewey \& Knoblich 2014, Saito et al 2015). At present time, there is insufficient evidence to justify the claim that the signals driving the intentional binding effects also drive agents' judgements about the voluntariness of movements.

It is clear that the above-mentioned studies employ an experimental approach where a movement, usually a button press, elicits some kind of external event. In some cases, this event resembles a delayed version of the actual movement performed using a delayed video signal, in other cases, the event is a planned auditory event. All existing studies (as far as we know) examine movements and their external consequences. We lack the experimental paradigms to study narrow SoA of movements without their external consequences.

Third, SoA has been studied in sensory attenuation experiments, where the sensations of consequences of a movement are diminished in strength when the movement is voluntarily performed. One of the underlying reasons for suggesting the idea of internal models in the central nervous system was the principle of sensory attenuation, which is the ability to diminish the sensory effect when one is the cause of the sensory effect, and, in particular, the ability to discriminate between externally generated sensory events and the sensory events one as an organism has produced. The sensory attenuation phenomenon has been shown in humans in tickling sensations (Blakemore et al 1998; Blakemore, Wolpert \& Frith, 2000) and force escalation (Shergill et al., 2003). The sensory attenuation effect has recently been studied in relation to SoA, for instance, in a study by Timm et al. (2016) that found that the N1 (negative deflection in the EEG 100 ms after a tone) component of an auditory event related potential (ERP) was not related to SoA but the P2 (positive deflection in the EEG $200 \mathrm{~ms}$ after a tone) component was. This was based on the argument that both the N1 and P2 amplitude of the ERP are reduced in size if the tone is self-generated, but if you shorten the interval between the action and the tone to be immediate, there was a reduction in subjective SoA as measured 
by a binary choice (yes or no, I did produce the tone) and the attenuation of the P2 amplitude was less strong.

The use of sensory attenuation in studies of SoA is problematic. Sensory attenuation is supposed to be the effect of a mechanism for cancelling out signals or at least for dampening them down. This makes it difficult to understand how the paradigm could be used to study the mechanism that is supposed to give rise to a positive SoA signal. If the SoA-mechanism function such that the better the match between prediction and actual sensory feedback, the stronger the signal for agency (the stronger the SoA), then such a SoA-mechanism would not have the properties of a sensory attenuation-mechanism, according to which the better the match, the weaker the signal. The only possible solution would be to incorporate an additional module that inverted the difference between the predicted and actual sensory feedback, and such modules have not been suggested.

If this brief review of the standard SoA paradigms gives us an adequate and representative picture of the field, then experimental SoA-research is confronted with a problem. Either standard experimental paradigms study the control and experience of distal consequences or they study sensory attenuation - and this goes both for explicit and implicit measures of SoA. Either way, there are problems. The consequence is that standard paradigms are ill suited for studying the narrow SoA for movements. This means that experimental work provides no evidence for or against the comparator model of SoA for movements. The fact that standard SoA-paradigms manipulate external events is a major obstacle to testing models of narrow SoA.

Summing the argument so far: Researchers have neglected to distinguish between narrow and broad SoA. Consequently, they have used the same comparator model to explain both narrow and broad SoA. This is problematic because the model is most plausible as a model of the narrow SoA for movements. The comparator model is not the only plausible model of narrow SoA. Specifically, narrow SoA could also be explained by a motor-signal model. Unfortunately, we do not currently have the appropriate experimental paradigms for testing models of narrow SoA. The standard SoAparadigms manipulate external events and, therefore, concern broad SoA. See Table 1.

[Insert Table 1 here]

\section{A new paradigm}

Assuming the truth of the comparator model, what predictions can we make about narrow SoA for movements? According to the comparator model, narrow SoA is the product of a match between 
predicted sensory feedback from making a movement and actual feedback from making a movement (see Figure $1 \mathrm{M} \& 1 \mathrm{~N}$ ). If we can experimentally create a match between predicted and actual feedback from movement, the comparator model would predict that participants would report SoA even if they did not actually move voluntarily. If an agent is confronted with many situations where the match between prediction and feedback from passive movement is very similar to a match between prediction and feedback from active movement, the comparator model would predict that the agent would start making discrimination mistakes. The purpose of our studies was to create illusion of narrow SoA, i.e. situations where participants would believe they had voluntarily performed a movement when in fact it was not themselves that had moved.

A number of critical issues in the design of these experiments require a discussion of two aspects of the comparator model. First, it is an open question when during the planning and execution of an action an efference copy is generated, and second, it is unclear whether corrections of movements necessarily requires SoA. From a purely theoretical point of view, the idea that the efference copy is a 1:1 copy of the motor command is present in the literature (e.g. fact box in Haggard, 2017, p. 201), but a number of electrophysiological studies have indicated that some motor signals sufficient to give rise to movements, i.e. motor commands, are not accompanied by efference copy signals (i.e. the signals which could give rise to the phenomena otherwise associated with efference copies, such as sensory suppression, intentional binding etc.).

There have been several attempts to elicit efference copies using non-invasive brain stimulation. If transcranial magnetic stimulation (TMS) is applied over the primary motor cortex (M1) it will elicit a movement of the target muscle (if applied with sufficient stimulation intensity). But for instance, Chronicle and Glover (2003) used TMS to elicit a movement that caused a needle to make a skin stimulation giving rise to a ticklish sensation. They showed that TMS induced movements did not elicit an efference copy that could be included in sensory suppression of the tickling sensation. Hence, the site of the motor command, i.e. M1, does not seem to be the locus of generating an efference copy sufficient to produce sensory suppression. Similar conclusions can be drawn from the original intentional binding studies (Haggard et al., 2002). Here TMS elicited muscle twitches and accompanying motor commands causing a sound did not give rise to the temporal binding of twitch and sound as a voluntary counterpart did. In a study of sensation of movements, Ellaway et al. (2004) came to a similar conclusion that efference copies were not generated in M1 after TMS stimulation. They raise the possibility that premotor cortex could be the site for the generation of the efference copy, i.e. a preparatory motor cortical area typically involved in execution of movements prior to the 
site of the motor command, i.e. M1. Similarly, Haggard and Whitford (2004) argued that the sensory suppression capability of efference copies was not issued from M1, but from another motor preparatory area, the supplementary motor area (SMA). Finally, Christensen et al (2010) showed that sensation of movement elicited by TMS stimulation over premotor cortex was unaffected by peripheral sensation block contrary to what was the case with M1 stimulation. They argued that premotor cortex, rather than the site of motor commands (M1), was responsible for generating efference copies.

In inclusion, there is reason to believe that motor commands from M1 are not sufficient to generate efference copy signals and that efference copies are generated upstream from M1. Rather than being simple copies of motor commands, there is evidence suggesting that efference copies are computed from goal representations in advance of the production of motor commands. The important point is that it is unclear exactly where and when in motor control process efference copies are produced. In our model presented in Figure 2, we propose that the efference copy is generated upstream to M1.

A number of studies have shown that fast online correction of movements can take place without the need of sensory feedback (Angel 1976; Desmurget et al., 1999; Pisella et al., 2000; Christensen et al., 2008). This is where the second comparator of the model proposed by Jeannerod (2006), and upon which we build our proposal, comes into play. The idea is that in the case of a shift in the online control of the movement, a correction can be issued through an early comparator. That is, a change in the desired state, or goal, leads to a mismatch between the desired state and the first issued forward model prediction. This enables fast online correction of the ongoing movement. In the study by Pisella et al. (2000), participants had difficulties in refraining from performing the corrections, despite being instructed not to do so. Our ambition was to create a similar situation in both our experiments.

We would like to produce a situation, that requires fast online correction of an ongoing movement. In addition, we will manipulate with sensory feedback, such that sensory feedback is in accordance with the initially issued movement. Therefore, we designed two experiments using a device that allow us to induce passive finger extension movements similar to their voluntary counterparts. The device will be referred to as the mechanical hand. The mechanical hand made it possible to have similar movements produced voluntarily or passively.

In the first experiment, we asked participants to perform a simple Go-Nogo task, and introduced passive movement using the mechanical hand. We asked participants to judge whether 
they had themselves performed the movement. We were particularly interested in the Nogo situations where participants were not supposed to perform a movement, but eventually in approximately $20 \%$ of the Nogo trials erroneously perform movements. If passive movements were applied to the Nogo trials, would participants be able to judge correctly whether a movement was an active or a passive movement?

In the second experiment, we asked participants to plan and execute finger extension movements of two different fingers according to predefined instructions. Depending on a cue signal, they had to prepare lifting either their index finger or thumb or both on a subsequent go signal. The Go signal either was in accordance with the prepared movement or required a change. Most of the times the Go signal was congruent with the cue signal but sometimes the participants had to change movement according to the Go signal. Introducing these changes, we would introduce conflicts that participants may or may not resolve by assigning agency to a movement that they initially had planned but which they did not perform. Thereby, we were in a situation where we could investigate the contribution planning of a movement makes to narrow SoA.

In experiment 1, our hypothesis was the following: The introduction of a passive movement of the finger in a Nogo trial, would potentially introduce situations where the participants report they have moved their finger but in reality, it is the mechanical hand that has moved the finger. We expect this to be the case because the participants make approximately $20 \%$ errors in these trials. Therefore, they are in a situation where they may or may not have made a mistake, and therefore prone to respond according to the passive displacement.

In experiment 2, our hypothesis was the following: The introduction of a passive movement in a situation where the participant has to change movement from the one that is planned as indicated by the cue signal, to the one that is instructed by the go signal, would potentially lead to situations where the participants erroneously report they have voluntarily moved the finger that was passively moved by the mechanical hand. This we believe will happen because the passive movement is in accordance with the initially planned movements that has to be corrected very fast.

\subsection{Experiment 1 - Go-Nogo}

\section{Participants}

Nine healthy participants were recruited for the study (5 females). All participant gave written informed consent before they entered the study. The study was approved by the local ethics committee of the capital region of Copenhagen (H-3-2013-198). 


\section{Methods}

Task

Participants were sitting on a chair in front of a table with their left hand resting in the mechanical hand device. The device was placed on the table. The index and middle finger was attached to the mechanical hand with Velcro straps. In front of the table was placed a green and a yellow LED. When the green LED was lit, the participant had to perform an index finger extension, as fast and as accurate as possibly. When the yellow LED was lit, the participants should omit the movement. The LEDs were placed next to each other, but in order to make the location of the green and yellow signal appear as close in space as possible, they were placed behind a piece of white paper, which made the lights appear in very similar locations. See Figure 3A.

Participants performed 2 rounds with 200 lights presented in each round, in total 400 lights. Approximately $80 \%$ of the lights were green Go lights, the remaining $20 \%$ were yellow Nogo lights. The order of the lights was presented randomly. After each light had been presented, the participants had to make or omit an index finger extension and afterwards report whether they themselves had performed a movement of the index finger. On all 400 trials the middle finger was passively moved by the mechanical hand. On $50 \%$ of the trials the index finger was moved passively at the approximate same time as when the participant would move the hand. The LEDs and the mechanical hand were controlled by a 1401 Micro III AD converter (Cambridge Electronic Design, Cambridge UK) using Signal (Cambridge Electronic Design, version 5.2) software.

[Insert Figure 3 here]

\section{Electromyography}

Electromyographic (EMG) activity was measured from the left forearm extensor muscle, m. extensor digitorum, using AMBU (AMBU A/S, Ballerup, Denmark) BlueSensor bipolar electrodes, a Neurolog (Digitimer Ltd., Letchworth Garden City, UK) EMG amplifier. The EMG signals were bandpass filtered at $5-500 \mathrm{~Hz}$ using the inbuilt filters of the Neurolog amplifier system. The EMG signals were digitized using a 1401 Micro III AD converter (Cambridge Electronic Design, Cambridge, UK) and processed in Signal (ver. 5.2, Cambridge Electronic Design, Cambridge, UK) software and Matlab (ver. R2015a, MathWorks, Natick MA, US) software (see Figure 3A). 
Analyses

The behavioural analyses consisted of identifying trials where participants had erroneously reported either that they had not moved during a Go trial or that they had moved during a Nogo trial. These trials were used to calculate an error fraction and d' in order to compare the conditions without and with passive movements of the index finger. Furthermore, all Nogo trials were inspected for the purpose of identifying abnormal EMG activity. This could either occur when the participants reported that they had not moved, but the EMG activity showed they had. Alternatively, it could happen in trials where participants reported that they had moved themselves but no EMG activity was present. We calculated error rates for the Go trials and Nogo trials with and without passive index finger movement and tested these using a two-way ANOVA with factors trial type (Go/Nogo) and passive movement (absent/present). Furthermore, we used signal detection theory and calculated the sensitivity index d'. We tested the difference in d' between the conditions with and without passive movements of the index finger using a paired t-test, we expected a decrease in d' with the introduction of passive movements of the index finger.

\section{Results}

Error rates \& sensitivity index d'

There was a trend towards a significant difference between the error rates in the Go and the Nogo trials $\left(\mathrm{p}=0.055, \mathrm{~F}_{1,32}=3.95\right)$ but no significant difference in error rates with and without passive movements $\left(\mathrm{p}=0.2537, \mathrm{~F}_{1,32}=1.35\right)$ and no significant interaction $(\mathrm{p}=0.4775, \mathrm{~F}=0.542)$ (See Figure 4A). The was no significant difference in d' between the condition with and without passive movements $\left(\mathrm{p}=0.1711, \mathrm{t}_{8}=1.5034\right)$ (See Figure 4B).

[Insert Figure 4 here]

\section{EMG and subjective reports}

When evaluating the divergence between objective measures of movement, i.e. EMG activity present during a task, and the subjective judgment of whether the participant had moved in the trial, we find the following results. In some cases, participants

- Report they have not moved in a go trial when they have moved as measured by EMG (77 movements in total in 9/9 participants, where 59 cases are from one participant) 
- Report they have not moved in Nogo trials when they have moved (27 movements in total in 6/9 participants)

- Report they have moved in Nogo trials when they have not (11 movements in total in $4 / 9$ participants)

- Report they have moved in go trials when they have not (2 movements in total in $1 / 9$ participants).

[Insert Table 2 here]

As is visible from Table 2, these discrepancies are not only due to the application of the passive movements, but also occur in trials without passive movements. (see Figs. 5 and 6).

[Insert Figure 5 here]

[Insert Figure 6 here]

\section{Conclusion of Experiment 1}

We did not find a convincing amount of trials where participants reported that they had moved voluntarily where they had not done so. This was only the case in a total of 11 trials in four participants based on measurements from a total of 3600 trials in 9 participants. We did find some deviations between the subjective experience of having moved and the objective measure of EMG activity. The most common deviation was that participants report that they had not moved in a Go trial when they had as measured by EMG. The second most common deviation was a report that they had not moved in NoGo trials when they had moved. Importantly, it appears that these discrepancies do not occur solely because passive movement are applied to the index finger (the finger participants are supposed to move on Go trials). In many cases, the same discrepancies occur in trials without passive movements being applied. 


\subsection{Experiment 2 - Hand control}

\section{Participants}

We recruited 16 healthy participants for experiment 2 . All participants gave written informed consent before they participated in the study. The study was approved by the local ethics committee of the capital region of Copenhagen (H-3-2013-198). Two participants were excluded from further analysis due to a technical problem, which caused all the trials to be mixed up in an order which was impossible to recreate.

\section{Methods}

Task

Each of the participants performed 540 finger extension movements of the index finger, thumb or both fingers. Each movement consisted of a preparation cue signal and a go signal indicating which finger(s) should be moved as quickly as possible. Both signals were LED lights above each finger (See Figure 3B). In $60 \%$ of the trials the cue and go signals were congruent. The distribution of trials was made such that in most cases there were congruency between the cue and the go signal, which would prime the participants to rely on the first cue signal rather than wait for the go signal and only rely on the go signal. The participants were encouraged to perform the movement as quickly as possible after the go signal.

In $71 \%$ of trials a mechanical displacement of either the index finger or thumb was introduced. In $44 \%$ of the displacements the passive movement was incompatible with the go signal. The choice of cue-go combinations was made such that there was an overweight of congruent combinations but still with a relatively large number of incongruent combinations with incompatible passive movement such that sufficient number of incongruent trials were made by each participant without having a very long experiment. The LEDs and the mechanical hand were controlled by a 1401 Micro III AD converter (Cambridge Electronic Design) using Signal (CED, version 5.2) software. The delays between the cue signal and go signal were randomly varied between $(500 \mathrm{~ms}$ and $2000 \mathrm{~ms}$, in steps of $500 \mathrm{~ms}$ ). The mechanical hand was activated $250 \mathrm{~ms}$ after the go signal was presented. After each movement participants subjectively reported which finger(s) they moved themselves and which finger(s) moved passively. The different cue- and go-signal combinations with additional mechanical placements are displayed in Figure 3B. The 21 combinations displayed in Figure 3B were categorized into six different combinations of Cue Signal - Go Signal - Passive displacement. The cue signal and the go signal could either be congruent or incongruent, which meant that it was either the same 
movement that was planned and performed, or it was a different combination of planned and performed movement. The passive displacement could also be compatible or incompatible with the performed movement, or there could be no passive movement (see Table 3 for the 6 different trial types).

[Insert Table 3 here]

The reduction of the 21 experimental conditions (shown in Figure 3B) into 6 trial types meant that planning of an index finger movement (based on the cue signal), which changed into a thumb movement on the go signal was a similar trial type as a trial where a thumb movement was planned, which was changed into an index finger movement, i.e. an InCon type.

\section{Electroencephalography and goniometer signals}

EEG recordings were obtained from 64 active electrodes (BioSemi, Amsterdam, NL) during the experiments. In addition, we measured finger displacements from a goniometer placed under the mechanical hand. The goniometer signals were digitized using a 1401 Micro III AD converter (Cambridge Electronic Design Cambridge, UK) and processed in the software Signal (version 5.2, Cambridge Electronic Design Cambridge, UK) software (see Figure 3A)

\section{Analyses}

The fraction of errors was based on the subjective reports and was calculated for each participant. We defined two types of errors, passive errors and voluntary errors. Passive errors were errors in determining which finger(s) was/were passively moved, and voluntary errors were errors with respect to which finger the participants voluntarily reported they moved relative to the instructed movement, i.e. when the participant had experienced that they moved differently than instructed. With respect to the voluntary errors, it turned out that the most frequent type of voluntary errors was a wrong movement according to the Go signal, which the participants were fully aware of. Alternative types of voluntary errors could be that the participant did not move, when they were supposed to, or it could be that they said they moved in one way, but actually moved in another way.

The error fractions were sorted into the 6 different trial types (see Table 3) with congruency or incongruence between cue and go signal and compatibility or incompatibility between the go signal and the passive movement or no passive movements. In order to test differences between the different 
trial types, the error fractions were transformed using an Arctan transformation, and then a two-way ANOVA was performed with the factors: Trial type (6 levels) and Error type (3 levels).

\section{EEG analyses}

The EEG data were initially pre-processed in EEGlab (Swartz Center for Computational Neuroscience, San Diego CA, USA), the data were average referenced, a band-pass filter between $0.25 \mathrm{~Hz}$ and $80 \mathrm{~Hz}$ was applied before an ICA analysis was employed in order to remove eye blink artefacts. Subsequently the data were transferred to the software SPM12 (http://www.fil.ion.ucl.ac.uk/spm/) for further analyses. Here the data were epoched either with respect to the different cue signals or the 6 different movement trial types. Finally, we calculated grand mean averages across the 14 participants for each of the cue types and each of the 6 trial types. The epoching was performed with respect to triggers indicating the onset time of the cue signal or the go signal.

\section{Results}

\section{Behavioural results}

The ANOVA of the error fraction revealed significant effects of movement type-combination $\left(\mathrm{F}_{5,10}=7.31, \mathrm{p}<0.001\right)$, significant effect of error-type $\left(\mathrm{F}_{2,10}=13.35, \mathrm{p}<0.001\right)$ and significant movement type-by-error type interaction $\left(\mathrm{F}_{10}=5.26, \mathrm{p}<0.001\right)$. Pair-wise comparisons revealed significant differences in the fraction of passive errors between the different movement types. However, the only type of movements that caused significantly more errors than any of the other movements was the InConCom. In this case, it was passive errors which were made more frequently (approximately $5.5 \%$ of the trials) when the cue and go signals were incompatible, i.e. where the participant had to perform another movement than the one planned due to the new go signal, and then subsequently the participant made more errors in determining which finger had been passively moved. However, contrary to our hypothesis, we did not find any situations where the amount of voluntary or combined voluntary and passive errors were increased. In particular, the situations with incompatible cue-go signals and incompatible passive movements and go signals revealed no such expected changes in error fractions (see Fig. 7).

[Insert Figure 7 here] 


\section{EEG results}

Due to the very few number of errors made as revealed by the behavioural analyses, we have not employed any EEG analyses of the differences between trials with and without specific types of errors. Here we only report data from the grand mean averages of the evoked responses following the 3 cue signals (Figure 8A) and from the 6 trial combinations of cue-go-passive movement types (Figure 8B) that were used in the experiment. The EEG analyses are depicted as scalp maps, where the colour coding indicates the scalp potential (red positive, blue negative, green 0) and these have then been plotted at different time points after the trigger indicating either cue signal or go signal. Results of the EEG analyses in Experiment 2 reveal that the passive movement is reflected in a right lateralized negative potential over central sensory-motor areas, suggesting that the passive movement is sensed or perceived as a signal separate from the movement of the hand alone.

[Insert Figure 8 here]

\subsection{Interim discussion}

Experiment 1 and 2 both failed to support our initial hypotheses. According to our first hypothesis, we expected that the introduction of a passive movement during a NoGo trial potentially could lead to more errors in judging whether the participant had performed a movement or not. Because participants at baseline during NoGo trials make approximately $20 \%$ errors, i.e. errors of commission, we hoped that the introduction of the passive movement would increase that error rate in terms of judged wrong movements. However, that was not the case. Participants made a similar amount of commission errors in the NoGo trials with and without passive displacement of the index finger.

In experiment 1, the introduction of the passive movements in the NoGo trials created no illusory SoA. As mentioned previously the spontaneous errors in the NoGo trials at baseline give a situation where it is likely that a participant may move (erroneously) in a trial where she is not supposed to move. According to the comparator model of SoA, this situation with a relatively high proportion of spontaneous errors could potentially give rise to an illusion of SoA. We found only very few examples of an experience of a movement despite the absence of a movement. The total number was very limited and occurred mainly in one participant. Furthermore, these experiences occurred more often in trials without passive movements. We did, however, observe a tendency towards denying having moved despite the presence of clear EMG activity in 6 of the 9 participants. We therefore believe the introduction of the passive displacement of the finger could have worked as 
an alternative explanation for the participants when they have done unwanted movement in the Nogo trials.

Our approach using passive movements of a finger could potentially be used in future studies with some critical improvements. Given the variability in reaction times, both within individual participants, as well as between participants, in both the Go-Nogo experiment and the cue-go experiment, slight timing mismatch between prediction of when to move and the passive displacement of the hand providing erroneous feedback may have hindered the illusion effect. We therefore suggest that monitoring of the actual movement could be used in order then to trigger or initiate the passive movements at the right time in comparison to when the participant initiates a voluntary movement. This would ensure that there is no discrepancy between the time of performing the movement and the passive displacement. However, this would not allow us to register the time at which a participant potentially would have moved, if the participant had not stopped moving during a No-go trial. We would therefore have to rely on brain-computer interfaces that potentially could decode the timing of when a participant will move, and whether or not the participant will move.

According to our second hypothesis, we expected an increase of errors with respect to voluntary judgments in the trials where participants initially planned one movement, but then were instructed at the time of go to make a different movement and were exposed to a passive movement of a finger that initially was planned to be moved. However, we did not see that the InConInCom trials lead to more voluntary or voluntary combined with passive errors compared with any of the other trial types. That is, participants almost never made the combined mistake of reporting having moved differently than they were instructed and making erroneous judgments of the passive movement. A critical factor could be that the fraction of congruent cue-go signals was too low (60\%) to make the participants fully rely on the cue signal for their preparation. By comparison, in the GoNogo design, there are $80 \%$ Go trials. In experiment 2, we aimed to balance a sufficient number of trials with incongruent cue-go signals and incompatible passive movements, in total 96/540 for each participant. In hindsight, too many InCon trials in the design might have caused participants not to rely on the cue signal alone. This design choice was made in order to have a sufficient number trials for the EEG analyses. An additional adjustment to the protocol could be to make the actual movement more automatized by not having a variable delay between the cue and the go signal.

In an ideal situation monitoring of EEG could reveal whether there is phenomenal difference between the situations with and without passive movements. At present, Experiment 2 reveals a qualitative difference in the EEG for trials with and without passive movements. This suggests that 
the passive movement may be experienced slightly different than similar voluntary movements. In future studies monitoring of EEG could be used to check whether there is a difference between trials with and without passive movements.

In terms of experimental design, the Go-Nogo is perhaps better suited to create situations of doubt, as to whether the participant experience SoA. This is mainly due to the higher baseline proportion of errors in the Nogo situation. However, for the purpose of creating illusory SoA experiences, it is perhaps a little counterintuitive to try and create that experience in the Nogo situation, where you are supposed not to move. In fact, the denial of having moved and the reluctance to ascribe a movement to the passive displacement highlights this ambiguity of the experimental setup.

\section{General discussion}

The first part of our argument provided us with three interim conclusions: 1) We should distinguish between narrow and broad SoA. The focus of this paper has been narrow SoA. 2) At least two possible models can explain narrow SoA. Narrow SoA could be driven by efferent motor signals (motor commands or efference copies) alone (see Figure 2A \& 2B) or it could be driven by a signal coming from a mechanism comparing forward model predictions with feedback from the movements (see Figure 1M). 3) At present, no obvious experimental paradigm seems to be suited for studying SoA for movements. Standard paradigms study either broad SoA or something different from SoA (for instance, sensory attenuation). Therefore, if narrow SoA for movements is going to be studied directly, new experimental paradigms are needed. In order to make progress with (2) and (3), we developed a new design to study narrow SoA. We created a situation where the match between sensory prediction and sensory feedback from passive movements should be very similar to situations in which the agent moves voluntarily. Given the comparator model, the prediction would be that in situations where the match between sensory predictions and feedback from passive movements is very similar to the match between sensory predictions and feedback from active movements, subjects would tend to make mistakes. They would tend to judge that passive movements were active movements.

The results do not support this prediction. We found no tendency to make mistakes of judgement. To be sure, the results are limited by being a null result. There are at least two ways of addressing our null results. The first way is in terms of likelihoods. Assuming the validity of our experiments, we can compare the likelihoods of the two competing models. If SoA is driven by motor 
signals, then the degree of match between predicted feedback and actual feedback from passive movement should not significantly influence SoA judgements. The probability of our results given this motor signal-model would be relatively high. By contrast, if SoA is driven by a signal from the comparator mechanism, the degree of match between predicted feedback and actual feedback from passive movement should influence SoA judgements. The probability of our results given the comparator model would be relatively low. On this interpretation of the null results, they weigh in favour of the motor signal model and weigh against the comparator model.

The second way of addressing the null results is by insisting that they tell us nothing. They could be explained by any number of factors, some of which we discussed above. Our contention is, however, that together with the theoretical arguments we have put forward, the results manage to raise an important challenge to the comparator model: why is it so difficult to create illusory SoA for movements? Further studies directly manipulating the actual movement are needed in order to elucidate the neural mechanisms that give rise to the narrow SoA for movements.

\section{Concluding remarks: the way forward}

We have shown that there is a need for a refinement of the definitions of SoA. Furthermore, even if SoA is defined narrowly in relation to control of movement, standard paradigms have used only manipulations of external consequences of the movement. Researchers have neglected to develop the necessary means to manipulate movements themselves. There is a need for new experimental paradigms that can address narrow SoA. If that goal is achieved, there may in addition be a need for a revision of the models that explain SoA. The preliminary results presented in this paper do not favour the comparator model of narrow SoA; rather, they weigh in favour of a motor signal-model. In future research, we need to develop the means to study the phenomenal experience of movements without the confounding presence of external consequences.

\section{Acknowledgements}

This work was supported by the Danish Independent Research Council - Humanities, grant 12126343 Phenomenal Consciousness and Cognitive Motor Control. In addition, MSC was funded by the Elsass Foundation. The funding sources had no involvement in the study design, data collection, data analysis, data interpretation or manuscript writing. 


\section{References}

Albus, J. S. (1971). A theory of cerebellar function. Mathematical Biosciences, 10(1-2), 25-61. Amassian, V., Cracco, R., \& Maccabee, P. (1989). A sense of movement elicited in paralyzed distal arm by focal magnetic coil stimulation of human motor cortex. Brain Research, 479(2), 355-360. Angel, R. (1976). Efference copy in control of movement. Neurology, 26(12), 1164-1168.

Balslev, D., Nielsen, F., Lund, T., Law, I., \& Paulson, O. (2006). Similar brain networks for detecting visuo-motor and visuo-proprioceptive synchrony. NeuroImage, 31(1), 308-312.

Balslev, D., Nielsen, F., Paulson, O., \& Law, I. (2005). Right temporoparietal cortex activation during visuo-proprioceptive conflict. Cerebral Cortex, 15(2), 166-169.

Blakemore, S. J., Wolpert, D., \& Frith, C. (2000). Why can't you tickle yourself? NeuroReport, 11(11), R11-6.

Blakemore, S., Frith, C., \& Wolpert, D. (1999). Spatio-temporal prediction modulates the perception of self-produced stimuli. Journal of Cognitive Neuroscience, 11(5), 551-559.

Blakemore, S., Wolpert, D., \& Frith, C. (1998). Central cancellation of self-produced tickle sensation. Nature Neuroscience, 1(7), 635-640.

Bridgeman, B. (2010). How the brain makes the world appear stable. I-Perception, 1(2), 69-72.

Caspar, E. A., Christensen, J. F., Cleeremans, A., \& Haggard, P. (2016). Coercion Changes the Sense of Agency in the Human Brain. Current Biology, 26(5), 585-592.

Carruthers, G. (2012). The case for the comparator model as an explanation of the sense of agency and its breakdowns. Consciousness and Cognition, 21(1), 30-45.

Christensen, M. S. \& Grünbaum, T. (2017) Sense of moving: Moving closer to the movement. In: Sensation of Movement. ed. Grünbaum T; Christensen MS. Abingdon, UK: Routledge: 64-84.

Christensen, M. S., Kristiansen, L., Rowe, J., \& Nielsen, J. (2008). Action-blindsight in healthy subjects after transcranial magnetic stimulation. Proceedings of the National Academy of Sciences, 105(4), 1353.

Christensen, M. S., Lundbye-Jensen, J., Grey, M. J., Vejlby, A. D., Belhage, B., \& Nielsen, J. B. (2010). Illusory Sensation of Movement Induced by Repetitive Transcranial Magnetic Stimulation. Plos One, 5(10).

Chronicle, E., \& Glover, J. (2003). A Ticklish Question: Does Magnetic Stimulation of the Primary Motor Cortex Give Rise to an “Efference copy?” Cortex, 39(1), 105-110. 
Daprati, E., Franck, N., Georgieff, N., Proust, J., Pacherie, E., Dalery, J., \& Jeannerod, M. (1997). Looking for the agent: an investigation into consciousness of action and self-consciousness in schizophrenic patients. Cognition, 65(1), 71-86.

David, N., Newen, A., \& Vogeley, K. (2008). The "sense of agency" and its underlying cognitive and neural mechanisms. Consciousness and Cognition, 17(2), 523-534.

Desmurget, M., Epstein, C. M., Turner, R. S., Prablanc, C., Alexander, G. E., \& Grafton, S. T. (1999). Role of the posterior parietal cortex in updating reaching movements to a visual target. Nature Neuroscience, 2(6), 563-567.

Desmurget, M., Reilly, K. T., Richard, N., Szathmari, A., Mottolese, C., \& Sirigu, A. (2009). Movement Intention After Parietal Cortex Stimulation in Humans. Science, 324(5928), 811-813.

Dewey, J. A., \& Knoblich, G. (2014). Do Implicit and Explicit Measures of the Sense of Agency Measure the Same Thing? PLoS One, 9(10), e110118.

Ellaway, P. H., Prochazka, A., Chan, M., \& Gauthier, M. J. (2004). The sense of movement elicited by transcranial magnetic stimulation in humans is due to sensory feedback. The Journal of Physiology, 556(Pt 2), 651-660.

Farrer, C., Franck, N., Georgieff, N., Frith, C. D., Decety, J., \& Jeannerod, M. (2003). Modulating the experience of agency: a positron emission tomography study. NeuroImage, 18(2), 324-333.

Feinberg, I. (1978). Efference copy and corollary discharge: implications for thinking and its disorders. Schizophrenia Bulletin, 4(4), 636-640.

Fink, G. R., Marshall, J. C., Halligan, P. W., Frith, C. D., Driver, J., Frackowiak, R. S. J., \& Dolan, R. J. (1999). The neural consequences of conflict between intention and the senses. Brain, 122, 497512.

Fourneret, P., \& Jeannerod, M. (1998). Limited conscious monitoring of motor performance in normal subjects. Neuropsychologia, 36(11), 1133-1140.

Fried, I., Katz, A., McCarthy, G., Sass, K. J., Williamson, P., Spencer, S. S., \& Spencer, D. D. (1991). Functional organization of human supplementary motor cortex studied by electrical stimulation. The Journal of Neuroscience, 11(11), 3656-3666.

Frith, C. (2015). The Cognitive Neuropsychology of Schizophrenia. (CLASSIC EDITION) (pp. 1165). Psychology Press.

Frith, C. D. (1987). The positive and negative symptoms of schizophrenia reflect impairments in the perception and initiation of action. Psychological Medicine, 17(3), 631-648. 
Frith, C. D., Blakemore, \& Wolpert, D. M. (2000). Abnormalities in the awareness and control of action. Philosophical Transactions of the Royal Society B-Biological Sciences, 355(1404), 17711788.

Gallagher, S. (2000). Philosophical conceptions of the self: implications for cognitive science. Trends in Cognitive Sciences, 4(1), 14-21.

Georgieff, N., \& Jeannerod, M. (1998). Beyond consciousness of external reality: a "who" system for consciousness of action and self-consciousness. Consciousness and Cognition, 7(3), 465-477.

Grünbaum, T. (2015). The feeling of agency hypothesis: a critique. Synthese, 192(10), 3313-3337.

Haggard, P. (2005). Conscious intention and motor cognition. Trends in Cognitive Sciences, 9(6), 290-295.

Haggard, P. (2017). Sense of agency in the human brain. Nature Reviews Neuroscience, 18(4), 196207.

Haggard, P., \& Chambon, V. (2012). Sense of agency. Current Biology, 22(10), R390-2.

Haggard, P., Clark, S., \& Kalogeras, J. (2002). Voluntary action and conscious awareness. Nature Neuroscience, 5(4), 382-385.

Haggard, P., \& Whitford, B. (2004). Supplementary motor area provides an efferent signal for sensory suppression. Brain Research Cognitive Brain Research, 19(1), 52-58.

Holst, E. V., \& Mittelstaedt, H. (1950). Das Reafferenzprinzip (Wechselwirkungen zwishcen Zentralnervensystem und Peripherie). Naturwissenschaften, 37(20), 464-476.

Imamizu, H., Kuroda, T., Yoshioka, T., \& Kawato, M. (2004). Functional Magnetic Resonance Imaging Examination of Two Modular Architectures for Switching Multiple Internal Models. The Journal of Neuroscience, 24(5), 1173-1181.

Ito, M. (1970). Neurophysiological aspects of the cerebellar motor control system. Int. J. Neurol., $7(2), 162-176$.

Jeannerod, M. (2006) Motor Cognition. Oxford University Press.

Jeannerod, M., Kennedy, H., \& Magnin, M. (1979). Corollary discharge: its possible implications in visual and oculomotor interactions. Neuropsychologia, 17(2), 241-258.

Johnstone, J. R., \& Mark, R. F. (1969). Evidence for efference copy for eye movements in fish. Comparative Biochemistry and Physiology, 30(5), 931-939.

Jordan, M. I., \& Rumelhart, D. E. (1992). Forward Models: Supervised Learning with a Distal Teacher. Cognitive Science, 16(3), 307-354 
Kawato, M., Furukawa, K., \& Suzuki, R. (1987). A hierarchical neural-network model for control and learning of voluntary movement. Biological Cybernetics, 57(3), 169-185.

Longo, M. R., \& Haggard, P. (2009). Sense of agency primes manual motor responses. Perception, 38(1), 69-78.

Lynn, M. T., Muhle-Karbe, P. S., Aarts, H., \& Brass, M. (2014). Priming determinist beliefs diminishes implicit (but not explicit) components of self-agency. Frontiers in Psychology, 5(DEC).

MacKay, D. M. (1951). Mindlike behaviour in artefacts. The British Journal for the Philosophy of Science.

MacKay, D. M. (1956). Towards an information-flow model of human behaviour. British Journal of Psychology, 47(1), 30-43.

MacKay, D. M. (1966). Cerebral Organzation and the Concious Control of Action. Brain and Conscious Experience Study Week September to October, of the Pontificia Academia Scientiarum, $422-445$.

MacKay, D. M., \& Mittelstaedt, H. (1974). Visual stability and motor control (reafference revisited). In Kybernetik und Bionik (pp. 1-10).

MacKay, D. M. (1978). The Dynamics of Perception. Cerebral Correlates of Conscious Experience INSERM Symposium No. 6: 56-68.

Marr, D. (1969). A theory of cerebellar cortex. The Journal of Physiology, 202(2), 437-470.

McCloskey, D. I. (1981). Corollary discharges: motor commands and perception. In: Handbook of Physiology (ed: ) (pp. 1415-).

Miall, R. C., \& Wolpert, D. M. (1996). Forward Models of Physiological Motor Control. Neural Networks, 9(8), 1265-1279.

Moore, J. W., Middleton, D., Haggard, P., \& Fletcher, P. C. (2012). Exploring implicit and explicit aspects of sense of agency. Consciousness and Cognition, 21(4), 1748-1753.

Moore, J. W., \& Obhi, S. S. (2012). Intentional binding and the sense of agency: A review. Consciousness and Cognition, 21(1), 546-561.

Moore, J. W., Ruge, D., Wenke, D., Rothwell, J., \& Haggard, P. (2010). Disrupting the experience of control in the human brain: pre-supplementary motor area contributes to the sense of agency. Proceedings Biological Sciences / the Royal Society, 277(1693), 2503-2509.

Mylopoulos, M. (2012). Evaluating the Case for the Low-Level Approach to Agentive Awareness. Philosophical Topics, 40(2), 103-127. 
Mylopoulos, M. (2017). Is there a bodily experience of agency? In Sensation of Movement. ed. Grünbaum T; Christensen MS. Abingdon, UK: Routledge: 42-56.

Nahab, F. B., Kundu, P., Gallea, C., Kakareka, J., Pursley, R., Pohida, T., et al. (2011). The Neural Processes Underlying Self-Agency. Cerebral Cortex, 21(1), 48-55.

Nielsen, T. (1963). Volition: A new experimental approach. Scandinavian Journal of Psychology, $4(1), 225-230$.

Pacherie, E. (2008). The phenomenology of action: a conceptual framework. Cognition, 107(1), 179217.

Pisella, L., Grea, H., Tilikete, C., Vighetto, A., Desmurget, M., Rode, G., et al. (2000). An “automatic pilot" for the hand in human posterior parietal cortex: toward reinterpreting optic ataxia. Nature Neuroscience, 3(7), 729-736.

Ritterband-Rosenbaum, A., Christensen, M. S., Kliim-Due, M., Petersen, L. Z., Rasmussen, B., \& Nielsen, J. B. (2011). Altered sense of Agency in children with spastic cerebral palsy. BMC Neurol. $11: 150$.

Ritterband-Rosenbaum, A., Christensen, M. S., \& Nielsen, J. B. (2012). Twenty weeks of computertraining improves sense of agency in children with spastic cerebral palsy. Res Dev Disabil. 33:12271234.

Ritterband-Rosenbaum, A., Karabanov, A. N., Christensen, M. S., \& Nielsen, J. B. (2014a). 10 Hz rTMS over right parietal cortex alters sense of agency during self-controlled movements. Front Hum Neurosci. 8:471.

Ritterband-Rosenbaum, A., Nielsen, J. B., \& Christensen, M. S. (2014). Sense of agency is related to gamma band coupling in an inferior parietal-preSMA circuitry. Front Hum Neurosci. 8:510.

Roland, P. E. (1978). Sensory feedback to the cerebral cortex during voluntary movement in man. The Behavioral and Brain Sciences, 1, 129-171.

Saito, N., Takahata, K., Murai, T., Takahashi, H. (2015). Discrepancy between explicit judgement of agency and implicit feeling of agency: Implications for sense of agency and its disorders, Consciousness \& Cognition 37, 1-7.

Sato, A., \& Yasuda, A. (2005). Illusion of sense of self-agency: discrepancy between the predicted and actual sensory consequences of actions modulates the sense of self-agency, but not the sense of self-ownership. Cognition, 94(3), 241-255.

Shepherd, J. (2016). Conscious action/zombie action. Noûs, 50(2), 419-444.

Shepherd, J. (2015). Conscious Control over Action. Mind \& Language, 30(3), 320-344. 
Shergill, S. S., Bays, P. M., Frith, C. D., \& Wolpert, D. M. (2003). Two eyes for an eye: the neuroscience of force escalation. Science (New York, NY), 301(5630), 187.

Spaniel, F., Tintera, J., Rydlo, J., Ibrahim, I., Kasparek, T., Horacek, J., ... \& Mikolas, P. (2015). Altered neural correlate of the self-agency experience in first-episode schizophrenia-spectrum patients: an fMRI study. Schizophrenia bulletin, 42(4), 916-925.

Sperry, R. W. (1950). Neural basis of the spontaneous optokinetic response produced by visual inversion. Journal of Comparative and Physiological Psychology, 43(6), 482-489.

Synofzik, M., Vosgerau, G., \& Newen, A. (2008). I move, therefore I am: A new theoretical framework to investigate agency and ownership. Consciousness and Cognition, 17(2), 411-424.

Synofzik, M., Vosgerau, G., \& Voss, M. (2013). The experience of agency: An interplay between prediction and postdiction. Frontiers in Psychology, 4(MAR), 1-8.

Timm, J., Schönwiesner, M., Schröger, E., \& Sanmiguel, I. (2016). Sensory suppression of brain responses to self-generated sounds is observed with and without the perception of agency. Cortex, $80,5-20$.

Tsakiris, M., \& Haggard, P. (2005). Experimenting with the acting self. Cognitive Neuropsychology, 22(3), 387-407.

Wen, W., Yamashita, A., \& Asama, H. (2016). Divided attention and processes underlying sense of agency. Frontiers in Psychology, 7(JAN), 1-8.

Wenke, D., Fleming, S. M., \& Haggard, P. (2010). Subliminal priming of actions influences sense of control over effects of action. Cognition, 115(1), 26-38.

Wolpert, D. M., Gharamani, Z., \& Jordan, M. I. (1995). An Internal Model for Sensorimotor Integration. Science (New York, NY), 269(5232), 1880-1882. 


\section{Tables}

\begin{tabular}{l|l|l} 
& Comparator model & Standard paradigms \\
\hline Narrow SoA & + & $\div$ \\
\hline Broad SoA & $\div$ & +
\end{tabular}

Table 1: Overview of the (mis)-alignment between what the comparator model tries to explain and what the standard paradigms can explain.

\begin{tabular}{|c|c|c|c|c|}
\hline Participant & $\begin{array}{l}\text { Report they have not moved } \\
\text { in a go ( } 320 \text { out of a total of } \\
400 \text { trials) trial when they } \\
\text { have moved as measured by } \\
\text { EMG }\end{array}$ & $\begin{array}{l}\text { Report they } \\
\text { have not moved } \\
\text { in Nogo } 80 \\
\text { trials out of a } \\
\text { total of } 400 \\
\text { trials) trials } \\
\text { when they have } \\
\text { moved }\end{array}$ & $\begin{array}{l}\text { Report they } \\
\text { have moved in } \\
\text { Nogo trials } \\
\text { when they have } \\
\text { not }\end{array}$ & $\begin{array}{l}\text { Report they } \\
\text { have moved in } \\
\text { go trials when } \\
\text { they have not }\end{array}$ \\
\hline 1 & $1 \mathrm{nP}$ & & & \\
\hline 2 & $1 \mathrm{wP}^{\S}$ & $\begin{array}{l}1 \mathrm{nP}^{\S}, 1 \mathrm{wP}^{\S}, 1 \\
\mathrm{nP}, 1 \mathrm{nP}^{\S}, 2 \mathrm{wP}^{\S}\end{array}$ & & \\
\hline 3 & $2 \mathrm{wP}$ & $5 \mathrm{nP}, 2 \mathrm{wP}$ & $1 \mathrm{wP}$ & $1 \mathrm{nP}, 1 \mathrm{wP}$ \\
\hline 4 & $1 \mathrm{nP}, 3 \mathrm{wP}$ & $5 \mathrm{nP}, 5 \mathrm{wP}$ & & \\
\hline 5 & $2 \mathrm{nP}, 1 \mathrm{wP}$ & $2 \mathrm{nP}$ & $5 \mathrm{nP}, 2 \mathrm{wP}$ & \\
\hline 6 & $2 \mathrm{wP}$ & & $1 \mathrm{nP}$ & \\
\hline 7 & $1 \mathrm{wP}$ & $1 \mathrm{wP}$ & & \\
\hline 8 & $2 \mathrm{nP}, 2 \mathrm{wP}$ & & $2 \mathrm{nP}$ & \\
\hline 9 & $5 \mathrm{nP}, 54 \mathrm{wP}$ & $1 \mathrm{wP}$ & & \\
\hline Total & $11 \mathrm{nP}, 66 \mathrm{wP}$ & $15 \mathrm{nP}, 12 \mathrm{wP}$ & $8 \mathrm{nP}, 3 \mathrm{wP}$ & $1 \mathrm{nP}, 1 \mathrm{wP}$ \\
\hline
\end{tabular}

Table 2: Experiment 1, overview of discrepancies between subjective reports of voluntary movement of the index finger and EMG activity measured form forearm finger extensor muscles. 
For each discrepancy type the number of trials of the specific type is indicated either without passive movement (nP) or with passive movement (wP). ${ }^{\S}$ EMG amplitude between $1 / 4$ and $1 / 2$ of EMG amplitudes during movements. Background EMG amplitude higher than in other trials, therefore difficult to determine with high certainty whether there was a movement or not.

\begin{tabular}{|c|c|c|c|c|c|}
\hline ConCom & ConInCom & $\underline{\text { InConCom }}$ & InConInCom & ConNoPas & InConNoPas \\
\hline $\mathrm{Cue}=\mathrm{Go}$ & $\mathrm{Cue}=\mathrm{Go}$ & $\mathrm{Cue} \neq \mathrm{Go}$ & Cue $\neq$ Go & $\mathrm{Cue}=\mathrm{Go}$ & $\mathrm{Cue} \neq \mathrm{Go}$ \\
\hline Go $=$ Passive & Go $\neq$ Passive & Go=Passive & Go $\neq$ Passive & Go & Go \\
\hline
\end{tabular}

Table 3: Experiment 2, overview of the six different combinations of congruent or incongruent cue and go signals with compatible, incompatible or no passive movement of the finger. 
Final draft. Please quote the published version: https://doi.org/10.1016/j.concog.2018.07.002

\section{Figure legends}


A

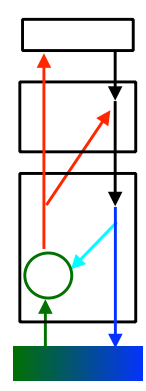

B
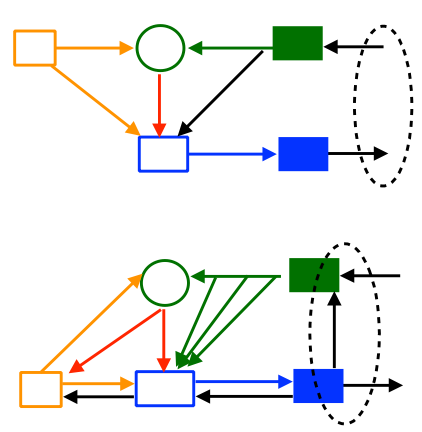

D

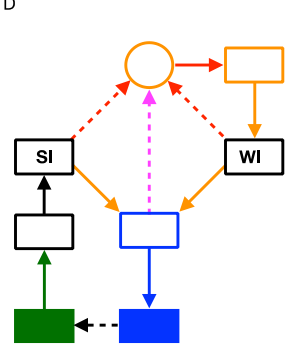

G

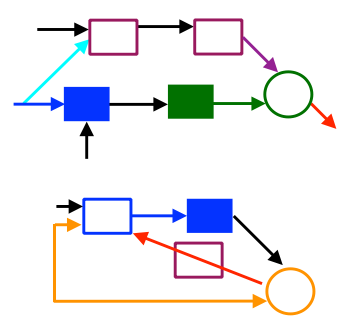

J

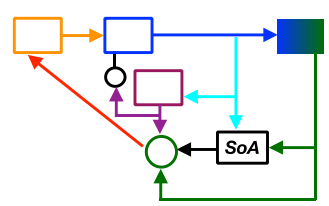

M

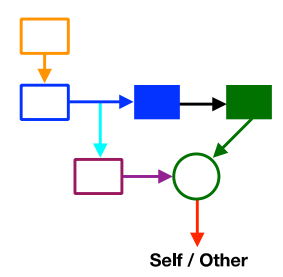

H

$\mathrm{N}$

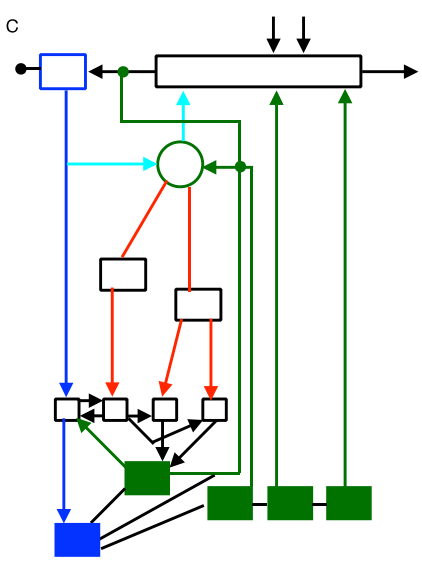

E
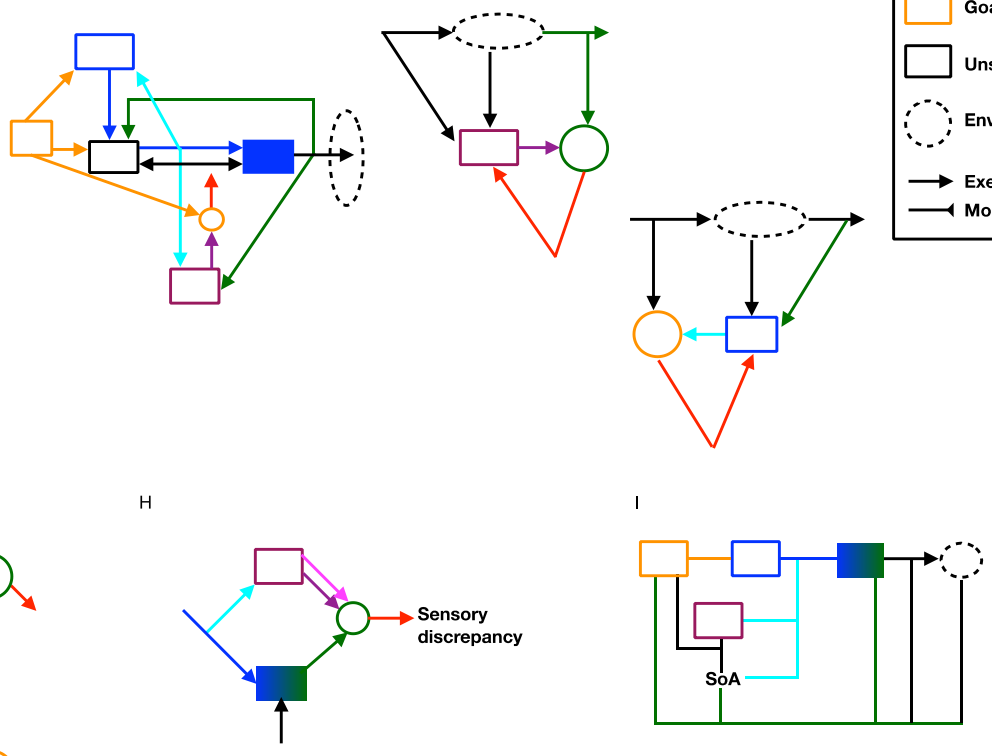

L
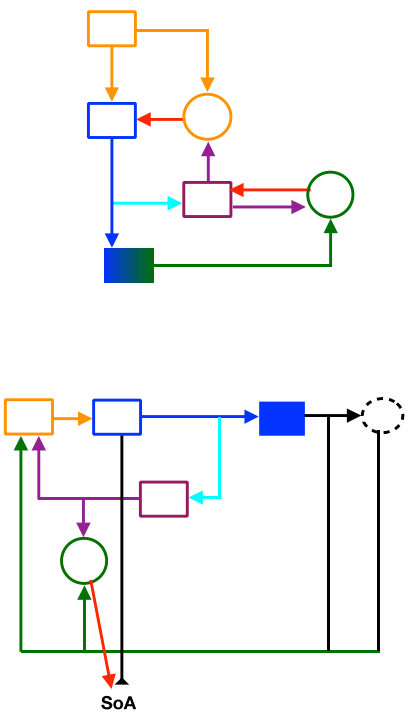

0

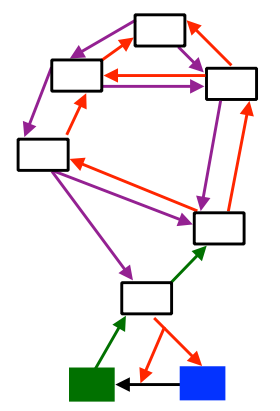

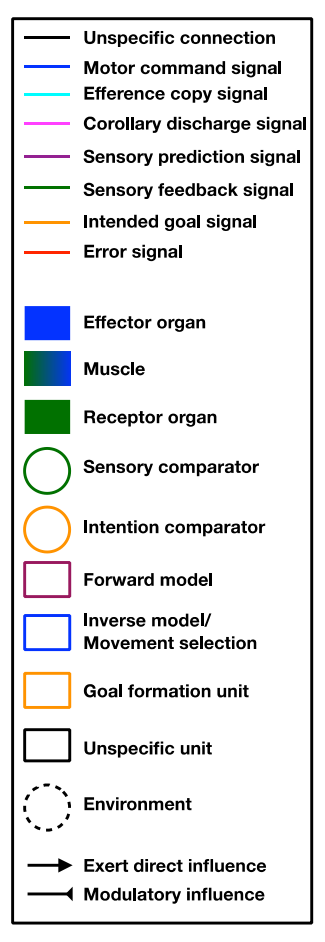

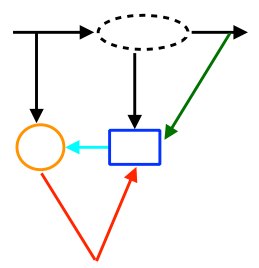

Modulatory influence 
Figure 1: The historical development of motor control models with a comparator element. A The efference copy principle proposed by Von Holst and Mittelsteadt (1950). B Various motor control principles for goal oriented behaviour proposed by MacKay in (1956) (top panel), (1966) (middle panel), and (1978) (bottom panel). C shows the feedforward hypothesis as presented by Roland (1978), note that both the input to and output from the sensory comparator is denoted an efference copy in the original paper. D The central monitoring theory by Frith (1987). The figure is a composite figure of two figures from the original paper. The sensory prediction feedback signal was named a corollary discharge in this paper, and therefore colour coded accordingly, but the feedback nature (according to Frith) indicated by the dotted line. SI: Sensory intention, WI: Willed intention. Dotted lines are called feedback, in the original proposal. E The internal model by Kawato et al (1987). F The forward model (left panel) and inverse model (right panel) for motor learning by Jordan and Rummelhart (1992). G The principles of sensory cancellation (top panel) and forward model for motor learning (bottom panel) by Miall \& Wolpert (1996). H Sensory cancellation principle describe by a comparator based on a forward model compared with sensory feedback by Blakemore et al (1999). Blakemore et al. call the signal from the forward model both a sensory prediction signal and a corollary discharge, hence the double arrow. I Description of sense of agency (SoA) based on a sensory prediction, an efference copy and sensory feedback by Haggard (2005), whether the individual effects on SoA are sufficient conditions or modulatory is not specified. J A proposal of an embodied agent by Tsakiri \& Haggard (2005), where a sensory bias is introduced that support SoA, but is not named SoA explicitly (therefore $S o A$ ). $\mathbf{K}$ A combined inverse and forward model for motor control with two separate comparators, an early and a late comparator as formulated by Jeannerod (2006). The first comparator for online correction and the second comparator for updates of the forward model. $\mathbf{L}$ The comparator principle and how it influences the feeling of ageny as described by Synofzik (2008), where the comparison between the predicted and actual feedback either gives rise to no sensory discrepancy or some sensory discrepancy. M The comparator principle described by David et al (2008), where absence of a discrepancy gives rise to 'self-agency' and presence of discrepancy gives rise to 'other-agency'. N SoA arise based on the comparison between predicted and actual feedback, large difference gives small SoA and no difference strengthen SoA signal. The signal from the inverse model exert a modulatory influence on SoA, but it cannot alone give rise to SoA. The model is proposed by Haggard \& Chambon (2012). O We have included the active inference model, as it is described in Adams et al (2013). Here it is interesting to note how the interpretations of predictive signals, motor commands and prediction error signals are reversed. The 
Active inference model of motor control described as a hierarchical generative model, where signals a mainly described as prediction signals and prediction error signals, here as formulated by Adams et al (2013). Each module compares signals and provide prediction errors for another module.

A

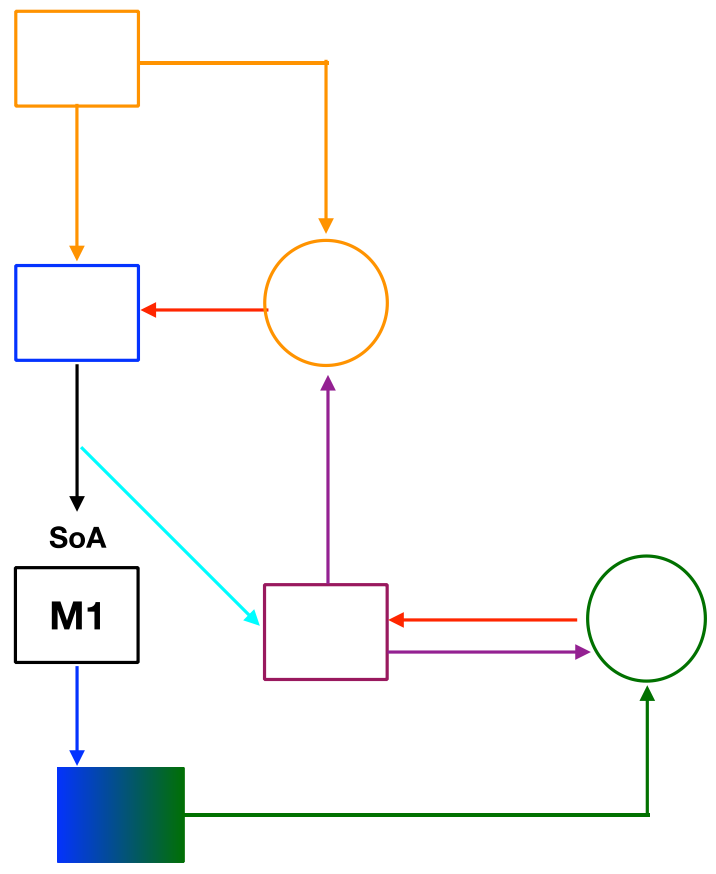

B

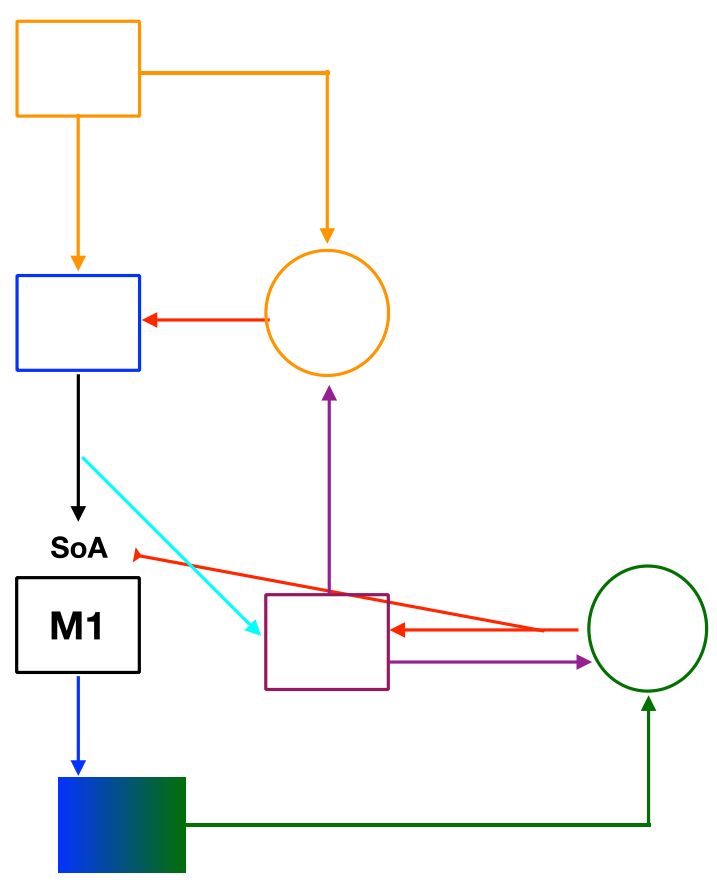

Figure 2: A a model of SoA where the motor signal (motor command or efference copy) is sufficient for SoA. B a model of SoA where an additional comparator output can modulate SoA. The model is an extension of the internal model proposed by Jeannerod (2006) presented in Figure 1K. See Figure 1 for notations used in this figure 

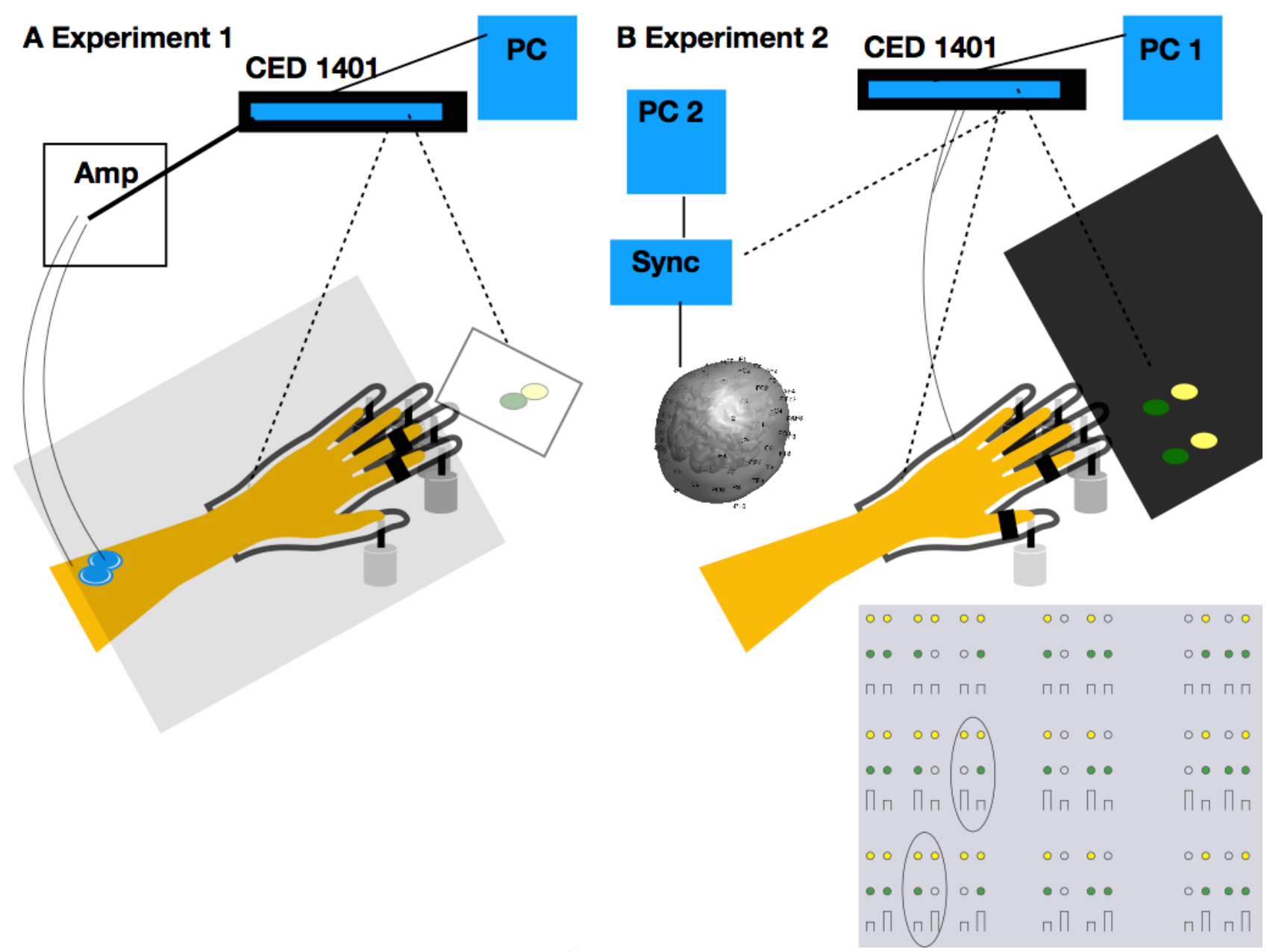

Figure $3 \mathrm{~A}$ shows a principle diagram of the setup used in Experiment 1, B shows a principle diagram of the setup used in Experiment 2. The fully draw lines from blue dots on the arms are EMG cables. The fully drawn line from the mechanical hand to the CED in Experiment 2, are goniometer signals. The dotted lines to the mechanical hand and the green and yellow dots are the signals that control the mechanical hand and the LEDs. The lower right corner displays the different cue (yellow) and go (green) signal combinations with additional information about the mechanical hand. The top row shows the trial types without mechanical displacement, the middle row shows the trials with mechanical displacement of the index finger and the bottom row with displacement of the thumb. The trial combinations highlighted in circles are the one's of primary experimental interest in Experiment 2. 
A

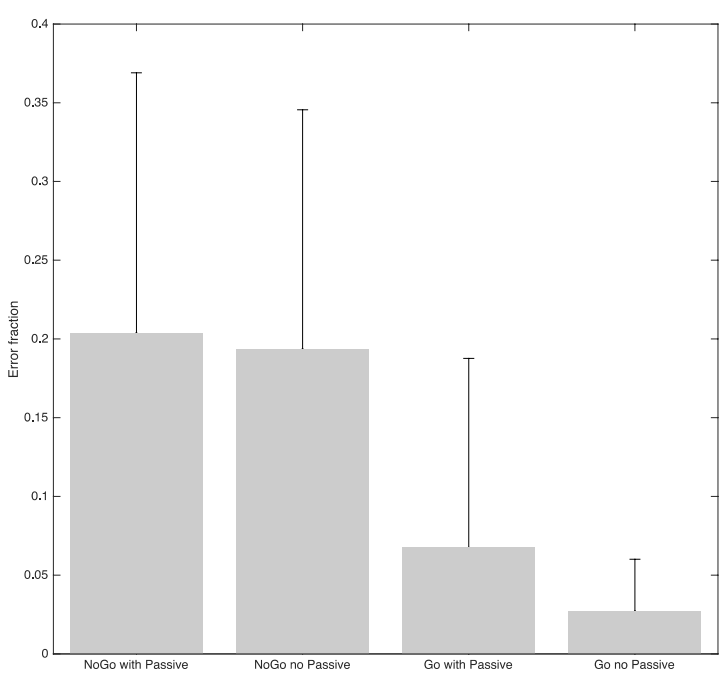

B

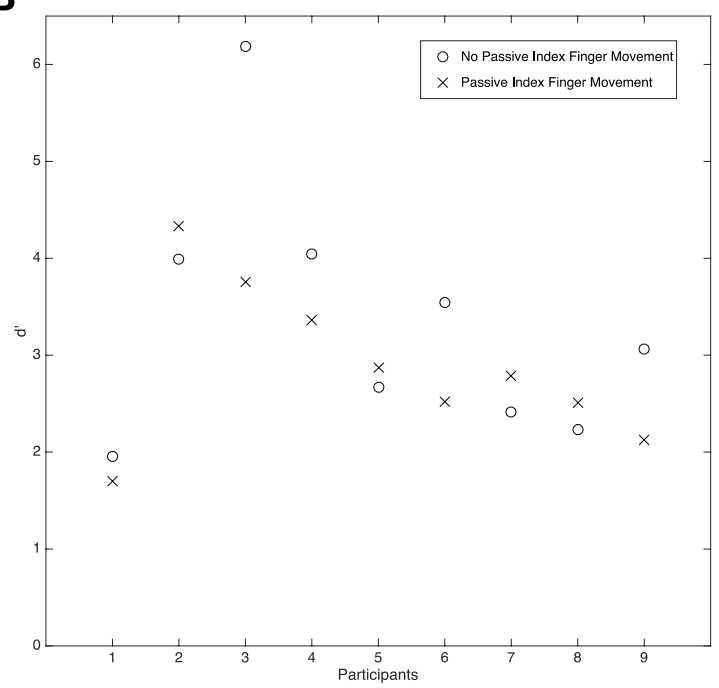

Figure 4: Experiment 1, A error rates in the Go-NoGo experiment. For the NoGo trials there are approximately $20 \%$ errors both without and with passive movements. For the Go trials the error rates are much lower. Error bars indicate standard deviations. B Sensitivity index d' calculated in all 9 participants for the trials with passive index finger movements and without passive finger movements. 

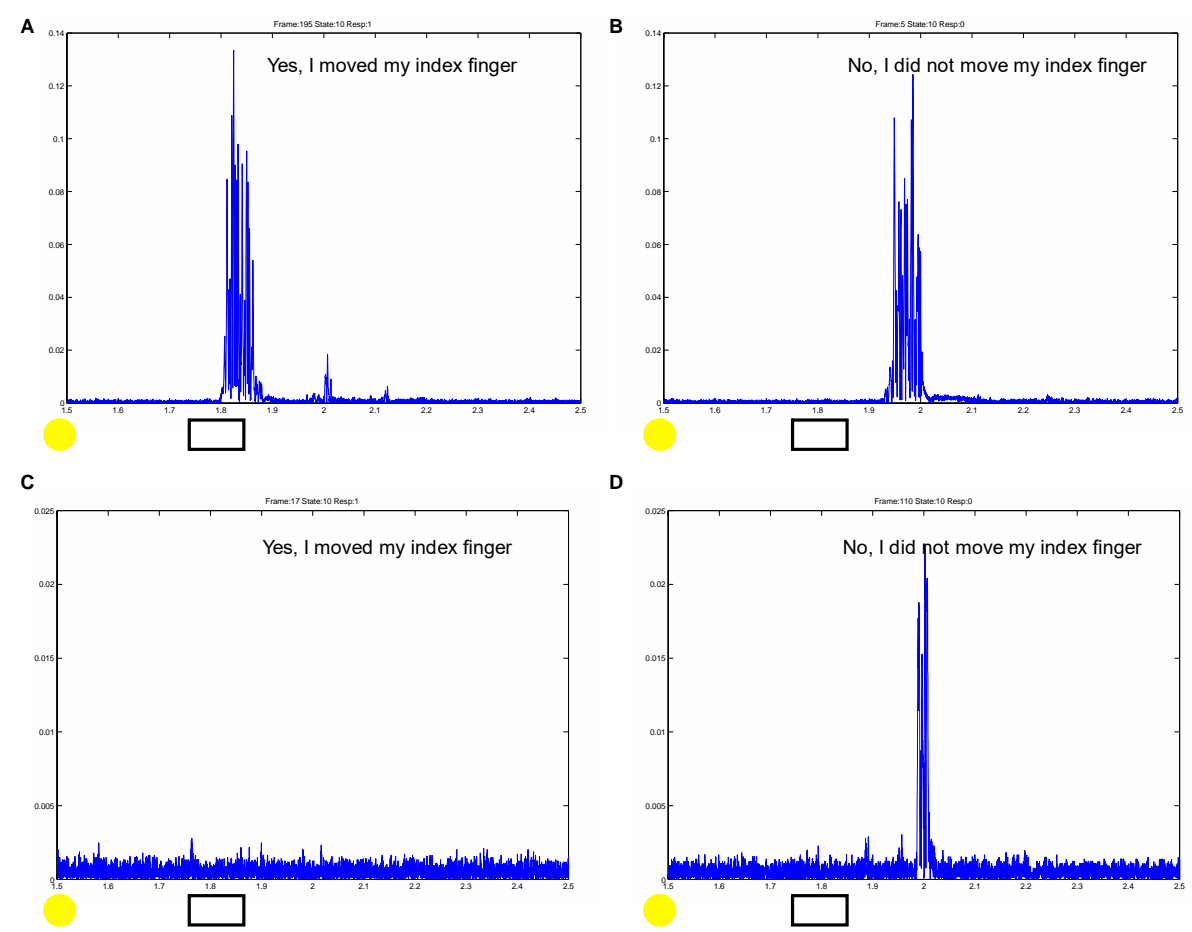

Figure 5: Experiment 1, EMG activity (measured in volts and rectified) measured for two NoGo trials with an example from Participant 4. The GoNo (yellow) signal was presented at time 1.5s, the passive movement (white rectangle) was applied at time $1.75 \mathrm{~s}$ for $100 \mathrm{~ms}$. (A) the participant report "Yes, I moved my index finger", (B) the participant report "No, I did not move my index finger". In both examples, there is a clear voluntary generated EMG burst. The example with EMG accompanying the report "Yes, I moved my index finger" was found in all 9 participants. The second example where participants denied they had moved, despite EMG activity was found in 6 participants in NoGo trials with passive movements in total 22 movements summed across all the 6 participants. (C \& D) EMG activity measured for two NoGo trials with an example from Participant 3. (C) The participant report "Yes I moved my index finger" despite no EMG activity present. This was in total present in 4 participants. In total across the 4 participants, this was found in 15 movements. (D) Example from participant 3, where the participant report "No, I did not move my index finger" despite clear EMG activity. (For the individual figure headlines, 'Frame:nnn' refers to trial number nnn, 'State:10' is a NoGo trial with an additional passive movement, 'Resp:1/0' refers to response from 
participant as to whether or not they moved their index finger). (For interpretation of the references to colour in this figure legend, the reader is referred to the web version of this article.)
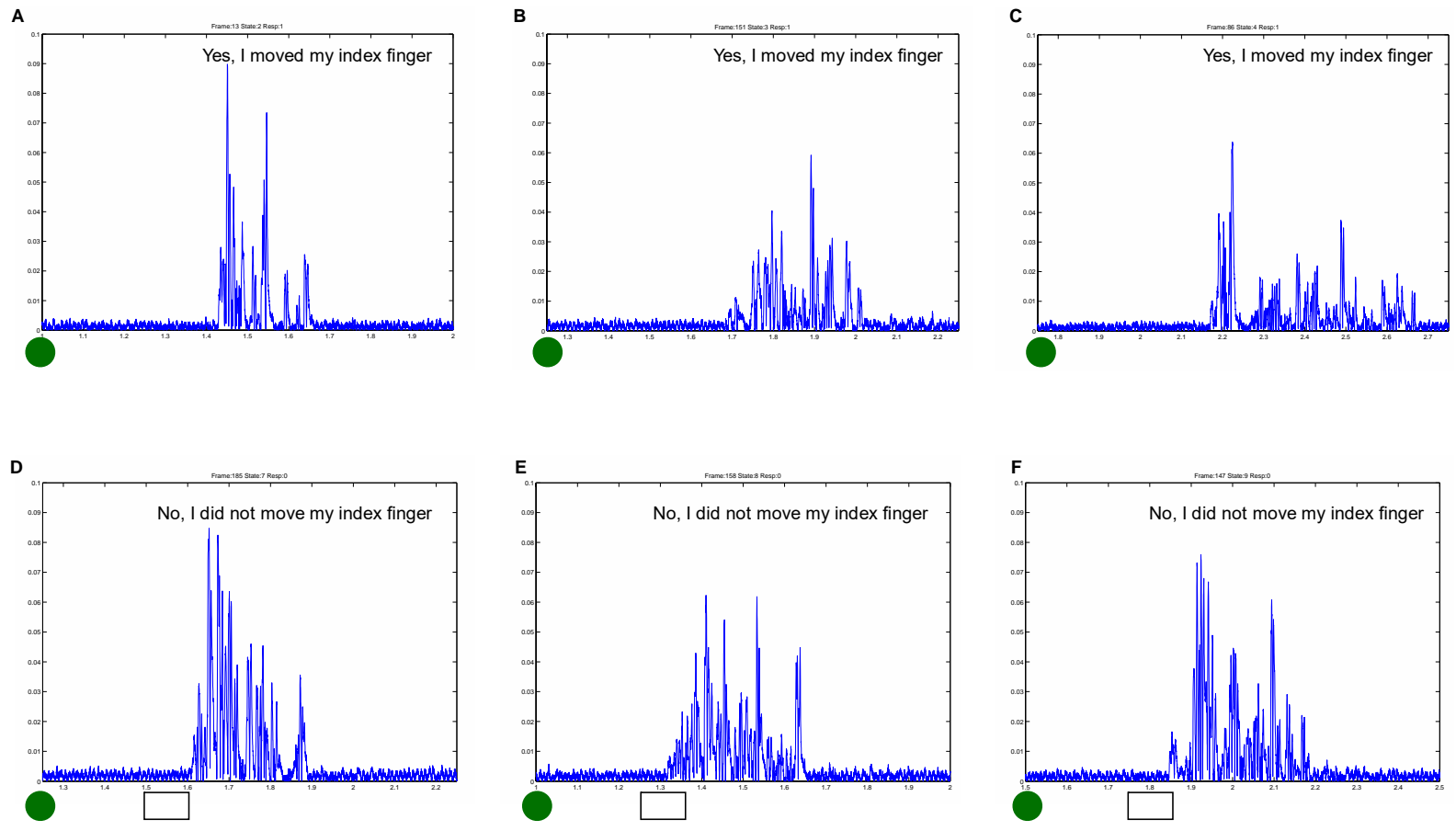

Figure 6: Experiment 1, EMG (measured in volts and rectified) examples from Participant 9. This participant showed an interesting pattern of denying having moved in 54 of 160 go trials with passive displacement throughout the experiment. A, B and C show Go trials where the participant report "Yes, I moved my index finger" with clear bursts of EMG. D, E and F show Go trials where the participant report "No, I did not move my index finger" despite clear bursts of EMG in the presence of passive displacement of the index finger. In a few (5) trials this particular participant also reported "No, I did not move my index finger" without the passive displacement. (For the individual figure headlines, 'Frame:nnn' refers to trial number nnn, 'State:2/3/4' are Go trials without passive displacement and 'State:7/8/9' are Go trials with passive displacement. 'Resp:1/0' refers to reponse from participant as to whether or not they moved their index finger). 


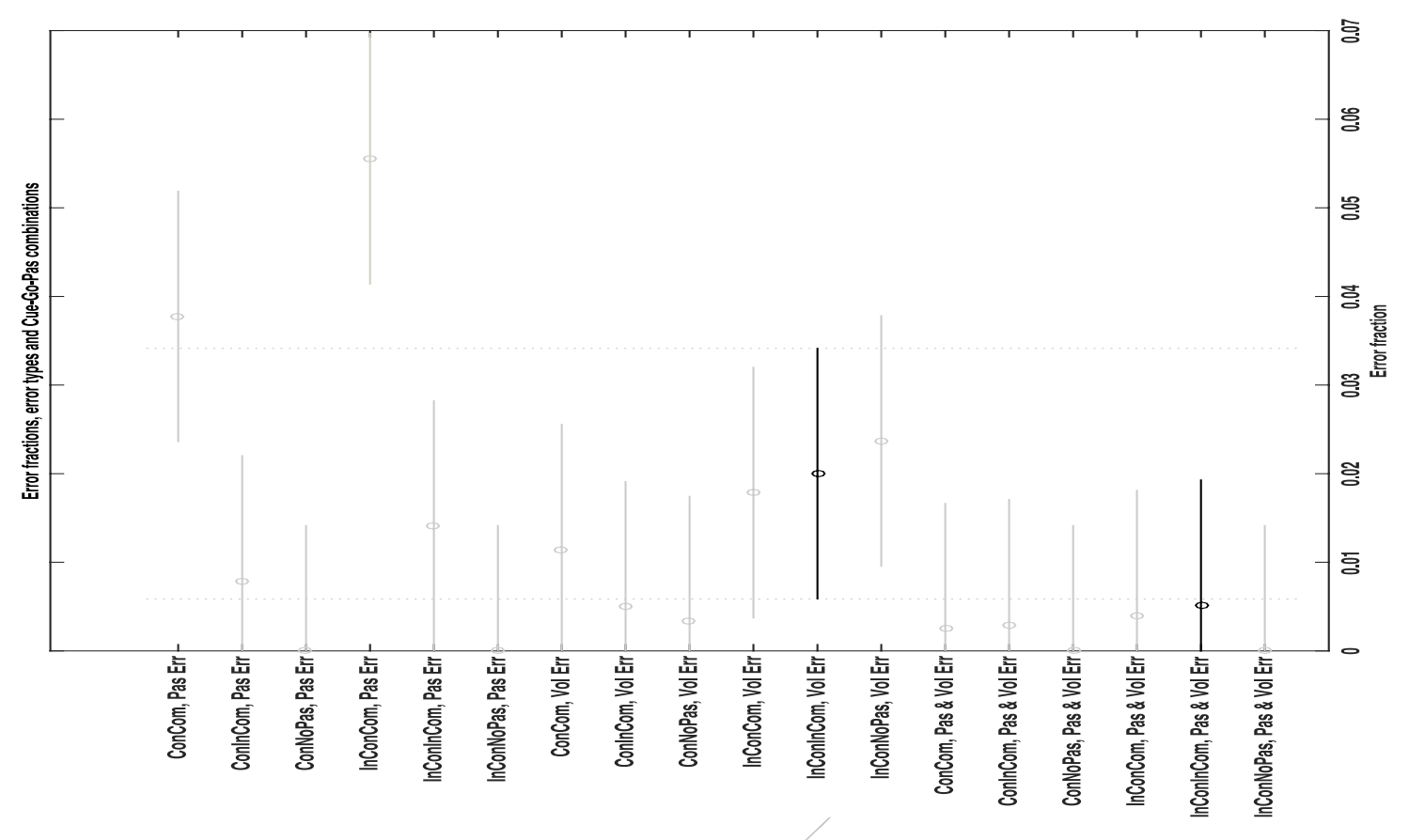

Figure 7: Experiment 2, error rates for the 6 types of cue-go-passive movement combinations sorted into the different types of error, i.e. passive errors, voluntary errors and the combined voluntary and passive error. The only type of experimental manipulation, that gives rise to significantly more errors are in congruent Cue-Go combinations with compatible passive movement (InConCom, Passive error). Here participants make more errors when they determine the passive displacement than in other experimental combinations (except ConCom). Our experimental manipulations of interest are marked in blue, those are the InConInCom trials and particularly whether more voluntary errors (or voluntary + passive error) were made. This does not appear to be the case. 


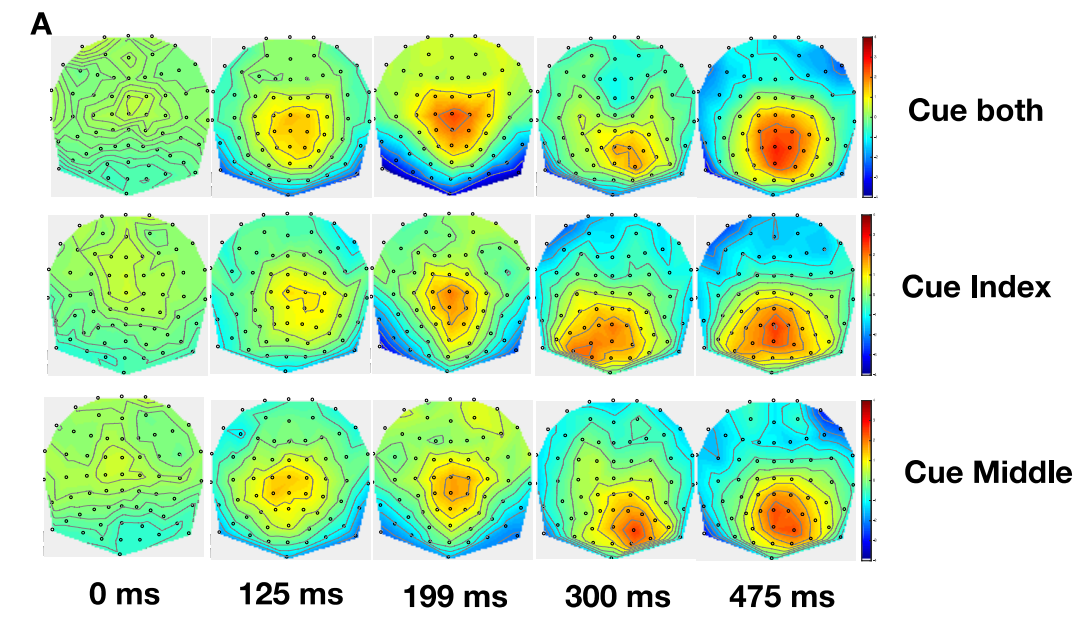

B
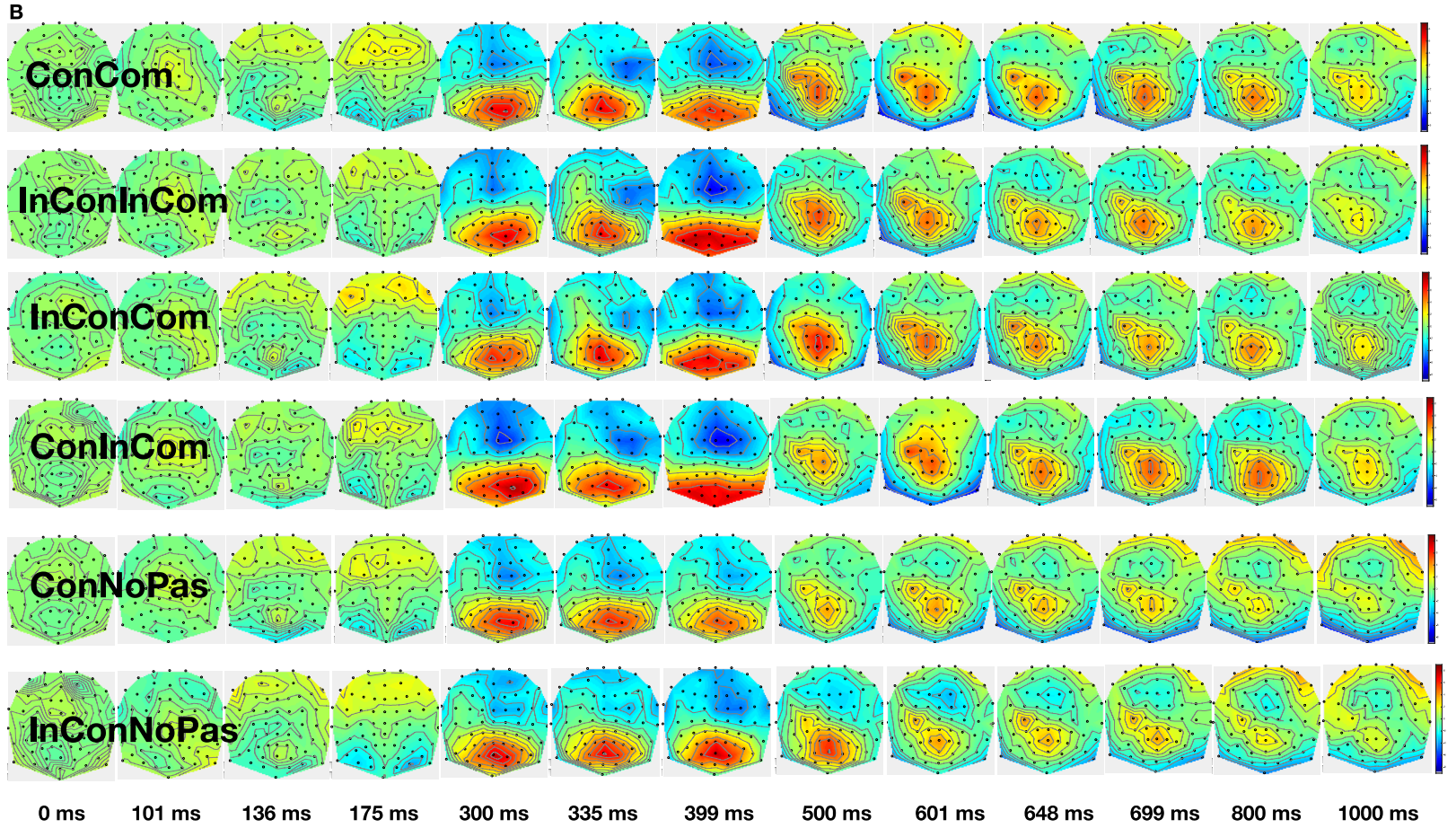
Figure 8 A Scalp maps of the electrical potentials for the three different cue types, indicating which movement the participant has to prepare. Cue Both indicate that both fingers should be extended when the go signal appears. Cue Index indicate that the index finger should be extended and Cue Middle, that the middle finger should be moved. The maps are displayed at 0, 125, 199, 300 and 475 $\mathrm{ms}$ after the cue signal is shown. B Scalp maps for the 6 different trials types used in the experiment. The only clear visual observation is that for the 4 trial types with passive movements (ConCom, InConInCom, InConCom, ConInCom) there is a negative frontal potential slightly right lateralised from approximately $335 \mathrm{~ms}-399 \mathrm{~ms}$ after the go signal was displayed. These four conditions also reveal a positive posterior signal present at 699 and $800 \mathrm{~ms}$ after the go signal, which is not present in the absence of the passive displacement. Colour bar indicate scalp potential in microV. 